\title{
A Kalman Filter Time Series Analysis method for InSAR
}

\author{
M. Dalaison ${ }^{1}$, R. Jolivet ${ }^{1,2}$ \\ ${ }^{1}$ École Normale Supérieure, PSL Université, CNRS U.M.R. 8538, Laboratoire de Géologie, Paris, France \\ ${ }^{2}$ Institut Universitaire de France, 1 rue Descartes, 75005 Paris, France
}

\section{Key Points:}

- Our data assimilation method for InSAR time series analysis allows for rapid update of pre-existing models with newly acquired data.

- Errors affecting the process are accounted for, so that each estimate is associated with its relevant uncertainty.

- We provide guidelines for the parametrization of our method. 


\section{Abstract}

Earth orbiting satellites, such as Sentinel 1A-B, build up an ever-growing set of synthetic aperture radar images of the ground. This conceptually allows for real-time monitoring of ground displacements using Interferometric Synthetic Aperture Radar (InSAR), notably in tectonically active regions such as fault zones or over volcanoes. We propose a Kalman filter (KF) for InSAR time series analysis, an efficient method to rapidly update pre-existing time series of displacement with data as they are made available, with limited computational cost. Our KF solves together for the evolution of phase change with time and for a parametrized model of ground deformation. Synthetic tests of our KF reveal exact agreement with the equivalent weighted least-squares solution and a convergence of descriptive model parameter after the assimilation of about one year of data. We include, the impact of sudden deformation events such as earthquakes or slow slip events on the time series of displacement. First tests of the KF on ENVISAT data over Mt Etna (Sicily) and Sentinel 1 data around the Chaman fault (Afghanistan, Pakistan) show precise $( \pm 0.05 \mathrm{~mm})$ retrieval of phase change when data are sufficient. Otherwise, the optimized parametrized model is used to forecast phase change. Good agreement is found with classic time series analysis solution and GPS-derived time-series. Accurate estimates are conditioned to the proper parametrization of errors so that models and observations can be combined with their respective uncertainties. This new tool is freely available to process ongoing InSAR time series.

\section{Introduction}

Since the 1990s, Interferometric Synthetic Aperture Radar (InSAR) has been used and optimized to measure ground deformation from satellite [e.g. Griffiths, 1995, Burgmann et al., 2000, Simons \& Rosen, 2015]. While first studies focused on temporally discrete, large amplitude events, such as earthquakes [e.g. Massonnet et al., 1993], recent geophysical applications rely on deriving the temporal evolution of deformation to capture the full spectrum of temporal behaviors, from short episodic deformation events [e.g. Lindsey et al., 2015, Rousset et al., 2016] to long-term, decadal trends [e.g. Grandin et al., 2012, Chaussard, Bürgmann, et al., 2014, Jolivet et al., 2015]. Examples include continuous monitoring of aquifers [e.g. Schmidt \& Bürgmann, 2003, López-Quiroz et al., 2009, Chaussard, Wdowinski, et al., 2014], volcanoes [e.g. Pritchard \& Simons, 2004, Biggs et al., 2014] slow moving landslides [e.g. Hilley et al., 2004, Scheingross et al., 2013, Tong 
\& Schmidt, 2016] or aseismic slip along active faults [e.g. Jolivet et al., 2013, Khoshmanesh \& Shirzaei, 2018].

Capturing the time evolution of ground displacement using InSAR is not direct and requires adequate processing of sets of interferograms [e.g. Simons \& Rosen, 2015]. An interferogram is the conjugate product of two Synthetic Aperture Radar (SAR) images. The corresponding interferometric phase directly records ground deformation between two passes of the satellite. Reconstructing the temporal evolution of the phase, hence ground deformation, through time should be straightforward. However, this procedure, called time series analysis, remains a challenge as interferograms are often affected by spatial and temporal decorrelation [e.g. Zebker \& Villasenor, 1992, Berardino et al., 2002, Simons \& Rosen, 2015]. Furthermore, the reconstructed phase is the combination of various sources of noise such as atmospheric and ionospheric delays as well as ground displacements. Part of the time series analysis procedure intends to separate these different contributions [e.g. Burgmann et al., 2000, Emardson et al., 2003, Agram \& Simons, 2015].

Two main approaches have been developed to perform time series analysis. First, Permanent or Persistent Scatterers techniques focus on a subset of reliable pixels with stable backscattering properties over time to perform spatial and temporal unwrapping [e.g. Ferretti et al., 2000, Hooper et al., 2007]. Second, Small Baseline Subset (SBAS) techniques rely on the redundancy of the network of interferograms to enhance spatial coverage and resolution [e.g. Berardino et al., 2002, Hetland et al., 2012]. In this paper, we focus on SBAS techniques.

Temporal increments of phase change are linked to interferograms by a set of linear equations. As we aim to reconstruct the evolution of phase with time from interferograms, we need to solve an inverse problem, which is usually done using classic least-squares [Schmidt \& Bürgmann, 2003, Usai, 2003, Agram et al., 2013] or singular value decomposition [Berardino et al., 2002, López-Quiroz et al., 2009, Jolivet et al., 2012]. Nowadays, the resolution, frequency and availability of SAR images grow dramatically thanks to recent launches of numerous SAR missions including the Sentinel 1A-1B (European Space Agency) or the ALOS 2 (Japan Aerospace Exploration Agency) missions [Elliott et al., 2016]. Future missions, such as Sentinel 1C-1D and NISAR (NASA, ISRO), will also lead to a growth in the amount of available data, ensuring long temporal coverage of deformation. Ex- 
isting SBAS techniques will inevitably become overwhelmed by the rapid accumulation of images. These methods require increasing computing power and memory, as the size of the inverse problem to solve grows with the quantity of observations. More importantly, acquisitions at a given time do not inform on the state of deformation at another given time if these epochs are not connected by interferograms. Processing the entire set of interferograms each time a new acquisition is performed is not only computationally expensive, but also not useful.

We, propose a method to sequentially update pre-existing multi-annual time series of InSAR data considering only the latest observations. We describe how to use data assimilation for the reconstruction of ground displacements using InSAR, including minimal computing time and little data storage. We derive the formulation of a Kalman filter for time series analysis, an approach analogous to least-squares in its assumptions and final solution [Kalman, 1960, Cohn, 1997]. As data assimilation methods require accurate estimation of errors at all steps, our method allows to investigate various sources of errors.

Kalman filtering is already widely used to build Global Navigation Satellite System (GNSS) time series [e.g. Hofmann-Wellenhof et al., 2012], as very frequent acquisition of small amount of data makes such filtering very relevant. Other application in geodesy include modeling of volcanic reservoir properties [e.g. Shirzaei \& Walter, 2010, Bato et al., 2018] or of fault slip at depth [e.g. Segall \& Matthews, 1997, Bekaert et al., 2016]. Most of these techniques are driven by time series of deformation in order to reconstruct the temporal evolution of a model describing the source of surface deformation. The aforementioned methods require the use of a time series analysis method in order to reconstruct surface displacements beforehand. Furthermore, although uncertainties are fundamental in any assimilation scheme, uncertainties are unfortunately not always correctly estimated [Agram \& Simons, 2015, Bekaert et al., 2016]. Here we provide a method to continuously and efficiently build InSAR time series from a stack of SAR interferograms and propagate associated uncertainties.

In the following, we detail time series analysis for InSAR and formulate explicitly the corresponding Kalman filter approach. We highlight the efficiency of our approach on two different regions subjected to volcanic and tectonic deformation. We first test our method on a time series of SAR acquisitions by the Envisat satellite between 2003 and 
2010 over the Etna volcano, in Sicily, around which several GPS stations enable us to derive local time series of ground deformation. We validate our approach against this independent set of data. We also use GPS data to assess the robustness of the uncertainties derived by our Kalman filter implementation. We then derive a time series of ground deformation using Sentinel 1 data between 2014 and 2018 over western Pakistan and southern Afghanistan. This region is poorly instrumented and no deformation time series are available for comparison with our approach. However, vegetation cover is scarce, hence interferometric coherence is good, and the Sentinel constellation has acquired a large amount of SAR images, allowing us to highlight the efficiency of our time series analysis method.

\section{A Kalman filter-based approach for times series analysis}

\subsection{Data description and formulation of the problem}

The phase of an interferogram is a differential measurement of the spatial and temporal change in the two way travel time of the Radar wave between the satellite and the ground. It is a direct measurement of the change in the apparent distance between the satellite and the ground, hence a function of ground deformation between two dates. Our goal is to reconstruct the evolution of the interferometric phase over time with respect to the first acquisition and to extract ground deformation from this time series. We work on each pixel independently from its neighbors [Berardino et al., 2002, Cavalié et al., 2007]. For a given pixel, the unwrapped phase of the interferogram between two dates at times $t_{i}$ and $t_{j}$ is

$$
\Phi_{i j}=\phi_{j}-\phi_{i}+\epsilon_{i j}
$$

where $\phi_{j}$ is the unwrapped phase at a time $t_{j}$ relative to the phase $\phi_{0}$ at time $t_{0}$, and $\epsilon_{i j}$ is the error associated with the potentially inaccurate unwrapping of the interferometric phase, with spatial filtering and with multi-looking (i.e. non-coherent phase averaging often used to enhance coherence) [Goldstein et al., 1988, De Zan et al., 2015, Agram \& Simons, 2015]. As interferograms connect multiple SAR acquisitions in time, we call a set of interferograms a network and $\epsilon_{i j}$ is often referred to as network misclosure [Doin et al., 2011]. Herein, the standard deviation of $\epsilon_{i j}$ will be noted $\sigma_{\epsilon}$, assuming it is common to all interferograms for simplicity. 
Moreover, for a single pixel, the network of interferograms is often incomplete as unwrapping of the phase is not always possible due to spatial and temporal variations of phase coherence. If the fringe rate is too high between neighboring pixels, it is not possible to derive the relative motion of these pixels from one to another, hence phase cannot be unwrapped [Goldstein et al., 1988]. Without connectivity, it is impossible to reconstruct a common phase history between temporally disconnected sets of interferograms. Various methods propose to derive a temporally parametrized model of the phase evolution, either assuming constant velocities between sub-networks [Berardino et al., 2002] or more complex ad hoc models [e.g. López-Quiroz et al., 2009, Jolivet et al., 2012, Hetland et al., 2012, Jolivet \& Simons, 2018].

Following the approach of López-Quiroz et al. [2009], we consider a parametrized function of time to describe the evolution of the interferometric phase. This function is the linear combination of a set of user-defined functions $f_{n}$ of time modulated by coefficients $a_{n}$, such as the interferometric phase $\phi_{i}$ at a time $t_{i}$ writes

$$
\phi_{i}=\sum_{n=1}^{N} a_{n} f_{n}\left(t_{i}\right)+\gamma_{i},
$$

where $\gamma_{i}$ is the error corresponding to mismodeling of the interferometric phase at time $t_{i}$, due to limitations of the functional model and decorrelation noise [Agram \& Simons, 2015]. Uncorrected atmospheric effects, such as turbulent and ionospheric delays, are the main contributions to $\gamma_{i}$ [e.g. Doin et al., 2011, Jolivet et al., 2014]. In the following, we assume that $\gamma_{i}$ is normally distributed with a zero mean and a standard deviation $\sigma_{\gamma}$, assumed constant with time for simplicity. Functions $f_{n}$ can be taken for instance as polynomial terms, Heaviside functions or periodic functions describing the time history of the interferometric phase.

Our goal is to solve both Equations 1 and 2 sequentially, whenever a new acquisition allows to compute new interferograms. We formulate an assimilation framework solving for the interferometric phase $\phi_{i}$ at each acquisition time $t_{i}$ and for the terms of the parametrized function $a_{k}$ and for the corresponding variances and covariances. 


\begin{tabular}{|c|c|c|c|}
\hline Symbol & Meaning & Structure & Shape \\
\hline $\mathbf{m}_{k}$ & state vector & {$\left[a_{0}, a_{1} \ldots, a_{L-1}, \phi_{0}, \ldots, \phi_{k}\right]$} & $(L+k+1)$ \\
\hline $\mathbf{d}_{k}$ & measurement vector & {$\left[\Phi_{f k}, \Phi_{g k}, \ldots, \Phi_{h k}\right]$} & $N$ \\
\hline $\mathbf{P}_{k}$ & state covariance & - & $(L+k+1) \times(L+k+1)$ \\
\hline $\mathbf{Q}_{k}$ & $\begin{array}{l}\text { process noise } \\
\text { covariance }\end{array}$ & $\begin{array}{l}\text { diagonal matrix* with last } \\
\text { element equal to }\left(\sigma_{\gamma}\right)^{2}\end{array}$ & $(L+k+1) \times(L+k+1)$ \\
\hline $\mathbf{R}_{k}$ & $\begin{array}{l}\text { observation noise } \\
\text { covariance }\end{array}$ & $\begin{array}{l}\text { diagonal matrix with }\left(\sigma_{\epsilon}\right)^{2} \\
\text { on the diagonal }\end{array}$ & $N \times N$ \\
\hline $\mathbf{A}_{k}$ & $\begin{array}{l}\text { state-transition } \\
\text { matrix }\end{array}$ & $\begin{array}{l}\text { identity matrix with additional } \\
\text { row using } a_{n} \text { to forecast } \phi_{k}\end{array}$ & $(L+k+1) \times(L+k)$ \\
\hline $\mathbf{H}_{k}$ & observation model & $\begin{array}{l}\text { pairs up the phases to build } \\
\text { the } \Phi_{i k} \text {. Contains } 0,1 \text { and }-1\end{array}$ & $N \times(L+k+1)$ \\
\hline
\end{tabular}

Table 1. Vectors and matrices used in the $k^{t h}$ Kalman filter iteration assimilating $N$ interferograms $\left(\Phi_{i k}\right)$ constructed with the acquisition at time $t_{k}$. At this given step, the filter (re)estimates the $N+1$ phases $\phi_{i, k}$ and the $L$ parameters $a_{n}$ of the linear descriptive model. $\left(^{*}\right)$ In our applications, diagonal elements of $\mathbf{Q}_{k}$ are zero except the last one, however, it may be useful to add non-zero systematic error in the first $L$ elements corresponding to $a_{n}$. See Appendix 7.1 for a detailed example of our KF matrices.

\subsection{Setup of the Kalman filter}

A Kalman filter $(\mathrm{KF})$ is an iterative procedure that allows to recover the least squares solution of an inverse problem by successively adding data. For a recent and detailed introduction to Kalman filtering, readers can refer to Evensen [2009] or Carrassi et al. [2018]. Data assimilation procedures propagate and update with newly acquired data the probability density function (PDF) of a given model. In a KF, the PDF of the model is a Gaussian distribution described by a state vector, $\mathbf{m}$, containing mean values for model parameters and the associated covariance matrix $\mathbf{P}$. Each time new data is acquired, a KF proceeds in two successive steps. 


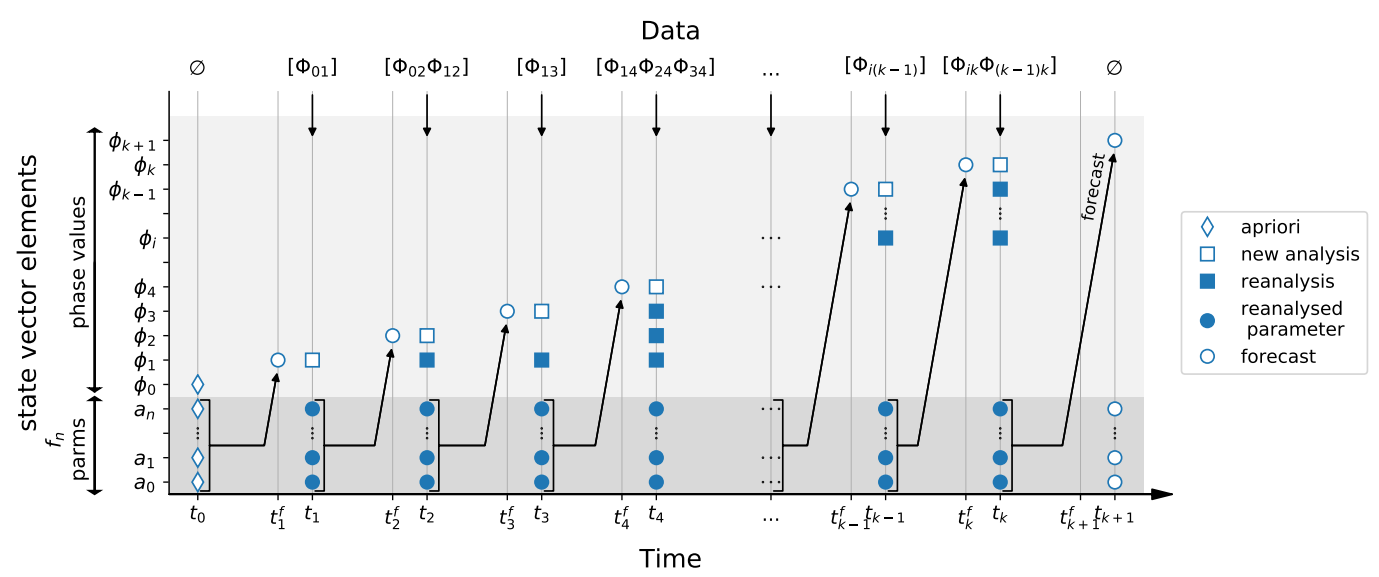

Figure 1. Scheme of the temporal evolution of the state vector as a function of assimilation time and available data. Markers highlight elements which are added or modified at a specific time-step. Additional values may be kept and stored for later reanalysis. The last step corresponds to the case when data are not available: the previous state vector is copied and the last phase is forecast using the functional description described by the parameters $a_{n}$ previously estimated.

First, at a given time $t_{k}$, we forecast the state vector $\mathbf{m}_{k}$ and its covariance matrix $\mathbf{P}_{k}$ using the state vector, $\mathbf{m}_{k-1}$, at step $k-1$. Second, we update this forecast with the information from data acquired at time $t_{k}$ in a step called analysis.

In practice, at a time $t_{k}$, the state vector $\mathbf{m}_{k}$ includes the reconstructed phase values and the coefficients of the parametrized function of time, $a_{n}$ (Figure 1). We initialize the framework with an a priori state vector, $\mathbf{m}_{0}$ and associated covariances, $\mathbf{P}_{0}$. This a priori reflects our state of knowledge on the different parameters before we input any data. Each time a new SAR image is acquired, we compute the interferograms connecting this last acquisition with previous ones, typically the four previous ones. Then, we use the functional form governed by the terms $a_{n}$ in the current state vector to forecast the phase at the time of the new acquisition. Afterwards, we analyze the forecast with the information of the incoming data to obtain the updated state vector.

Following the marginalization rule, the forecast state vector $\mathbf{m}_{k}^{f}$ and its covariance $\mathbf{P}_{k}^{f}$ are given by

$$
\mathbf{m}_{k}^{f}=\mathbf{A}_{k} \mathbf{m}_{k-1} \quad \text { and } \quad \mathbf{P}_{k}^{f}=\mathbf{A}_{k} \mathbf{P}_{k-1} \mathbf{A}_{k}^{T}+\mathbf{Q}_{k} \quad \forall k \in[1 ; M]
$$


where $\mathbf{A}_{k}$ is the state-transition matrix and $\mathbf{Q}_{k}$ the process noise covariance (see Table 1 for variable description). As illustrated in Figure $1, \mathbf{A}_{k}$ applied to $\mathbf{m}_{k-1}$ computes the forecast for $\mathbf{m}_{k}$ based on the latest parametric description of the time series given by $a_{n}$ at time $t_{k-1}$ (Equation 2). $\mathbf{A}_{k}$ is a matrix representation of the forecast equations. In practive, phase terms of previous acquisitions and functional parameters are kept constant while $\phi_{k}^{f}$ is computed using Equation 2. Because $\mathbf{A}_{k}$ is of rectangular shape, $m_{k}^{f}$ is simply $m_{k-1}$ augmented with the forecast phase value of the most recent acquisition. The corresponding covariance, $\mathbf{P}_{k}^{f}$, depends on parameter uncertainties and systematic noise included in $\mathbf{Q}_{k}$. Systematic noise in $\mathbf{Q}_{k}$ represents our level of confidence in an imperfect forecast.

We then update the state and its covariance using the available data $\mathbf{d}_{k}$ at time $t_{k}$ applying Bayes' rule, so that

$$
\mathbf{m}_{k}=\mathbf{m}_{k}^{f}+\mathbf{P}_{k}^{f} \mathbf{H}_{k}^{T}\left(\mathbf{R}_{k}+\mathbf{H}_{k} \mathbf{P}_{k}^{f} \mathbf{H}_{k}^{T}\right)^{-1}\left(\mathbf{d}_{k}-\mathbf{H}_{k} \mathbf{m}_{k}^{f}\right)
$$

where $\mathbf{R}_{k}$ is the observation noise covariance, and $\mathbf{H}_{k}$ the observation model. $\mathbf{H}_{k}$ is effectively the operator predicting interferograms from the state vector $\mathbf{m}_{k} . \mathbf{R}_{k}$ describes our confidence in the observation model, $\mathbf{H}_{k}$, for the data $\mathbf{d}_{k}$ (hence, $\mathbf{R}_{k}$ describes the statistics of phase misclosure, here assumed normally distributed). The analyzed variance covariance matrix, $\mathbf{P}_{k}$, is

$$
\mathbf{P}_{k}=\mathbf{P}_{k}^{f}-\mathbf{P}_{k}^{f} \mathbf{H}_{k}^{T}\left(\mathbf{R}_{k}+\mathbf{H}_{k} \mathbf{P}_{k}^{f} \mathbf{H}_{k}^{T}\right)^{-1} \mathbf{H}_{k} \mathbf{P}_{k}^{f} .
$$

The term $\mathbf{P}_{k}^{f} \mathbf{H}_{k}^{T}\left(\mathbf{R}_{k}+\mathbf{H}_{k} \mathbf{P}_{k}^{f} \mathbf{H}_{k}^{T}\right)^{-1}$ is often referred to as the Kalman gain as it quantifies how much the predicted state "has to gain" from the difference between observed and predicted data, $\mathbf{d}_{k}-\mathbf{H}_{k} \mathbf{m}_{k}^{f}$, called residual or innovation. It also modulates the information transitioning from the covariance in the model space $\mathbf{H}_{k} \mathbf{P}_{k}^{f}$ to the covariance of the analysis. For a practical example of our KF, see the the explicit formulation in Appendix 7.1. Note that Equation 4 is the generalized least squares solution of a linear inverse problem [e.g. Tarantola, 2005].

Because of observations equations, there is a need to keep previous estimates of phases in $\mathbf{m}_{k}$, whenever they are connected by interferograms used in the analysis step, in order to update phases $\phi_{i}(i<k)$ for all $\Phi_{i k}$ in $\mathbf{d}_{k}$ (Eqn. 1 and Fig. 1). For instance, if the data contains interferograms $\Phi_{a k}, \Phi_{b k}$ and $\Phi_{c k}$ we will forecast and analyze $\phi_{k}$ and 
re-analyze $\phi_{a}, \phi_{b}$ and $\phi_{c}$ using past and current observations (Figure 1). This is essential to keep improving phase estimates taking advantage of the redundancy of information from all interferograms and, thus, limit the propagation of errors over time. Formally, the re-analysis of past estimates with future data implies that the Kalman filter formulated above is effectively a Kalman smoother [Cohn et al., 1994, Cosme et al., 2012].

\subsection{Configuring parameters}

The algorithm requires user-based choices for the parametrization of the functional form and for the various covariances on a case-by-case basis. First, we have to chose a parametrization for the functional form used to derive the forecast. This choice is based on our knowledge of deformation and simplicity of the model should be favored over precision to prevent overfitting.

Second, we need to estimate the typical standard deviation of mismodeling $\sigma_{\gamma}$ for all time steps and of interferometric network misclosure $\sigma_{\epsilon}$ for all interferograms. $\sigma_{\epsilon}$ comes in the construction of $\mathbf{R}$ because it quantifies the error between our data (interferograms) and what we are looking for (the relative phase values). It effectively acts as a regularization term when computing the Kalman gain (Table 1 and Equations 4,5). As underlined by Doin et al. [2011], covariance terms in $\mathbf{R}$ are null because $\epsilon_{i j}$ is specific to each interferogram $\Phi_{i j}$, independently of the common acquisitions $\phi_{i, j}$. Regarding $\sigma_{\gamma}$, it depends on both the simplicity of the chosen functional form and on the amplitude of unexpected atmospheric perturbations of the interferometric delay. It is directly fed into the process noise variance-covariance matrix, $\mathbf{Q}$, since it controls the flexibility given to the process for phase values to be different from those predicted by the chosen functional form. Typically, $\sigma_{\epsilon}$ should be small with respect to $\sigma_{\gamma}$ because we have greater trust in the interferogram construction (Equation 1) than in the functional description of the deformation (Equation 2).

Third, we must quantify the a priori mean and standard deviation of functional model coefficients $a_{n}$ within the initial state vector $\mathbf{m}_{0}$ and covariance $\mathbf{P}_{0}$. These values directly control the amplitude of the possible values for model coefficients in the analyzed state vector, and, thus, directly affect the quality of the filter's forecast. One needs to chose large enough variances with a realistic a priori state vector, so that the natural spread of the variable is within one standard deviation of its mean. 
Fourth, we can optionally add some systematic error to the parameters of the functional representation, $a_{n}$ in the $L$ first elements of the diagonal of $\mathbf{Q}$ in order to slow down their convergence with assimilation steps. Such noise addition introduces some plasticity in the description of deformation (see Appendix 7.1). We discuss real case examples below to illustrate the influence of the different parameters and define a quantitative guideline for parametrization in Section 4.1.

\section{Applications of the Kalman filter}

\subsection{Synthetic tests}

\subsubsection{Reference case setup}

In order to asses how well the Kalman filter $(\mathrm{KF})$ retrieves known parameters, we generate a synthetic set of InSAR data combining synthetic signal of tectonic deformation and atmospheric noise. We assess the influence of the choice of parameters and of the design of covariance matrices to validate the approach.

We build a two dimensional, time varying, field of phase change typical of what is expected in a region crossed by a major tectonic fault. We design a synthetic acquisition planning considering a 3 years observation period with acquisitions every 12 days; similarly to what is expected for recent satellite constellations such as Sentinel 1. For each of these synthetic acquisitions, we compute synthetic unwrapped interferograms with the three preceding acquisitions using Equation 1.

We simulate the contributions of tectonic plate motion and shear due to interseismic loading along a fault (i.e slow, persistent deformation of a few $\mathrm{cm} / \mathrm{yr}$ ), between blocks moving at $4 \mathrm{~cm} / \mathrm{yr}$ with respect to each other. We add to the time series the surface displacements due to a typical earthquake (i.e. a discrete, large amplitude deformation event of several $\mathrm{cm}$ ). The modeled earthquake occurs on day 500 of the time series and induces a maximum of $15 \mathrm{~cm}$ of displacement. In addition, we consider the case of a slow transient slip event occurring on the same fault (i.e. episodic, medium amplitude deformation spanning multiple acquisitions in time). This slow slip event has a temporal footprint governed by an integrated spline function of 100-day-width centered on day 210 of the time series, with a maximum cumulative displacement of $10 \mathrm{~cm}$. Epicenters of both events are shown on Figure 2. All synthetic displacements are generated considering dislocations embedded in an elastic homogeneous semi-infinite halfspace [Okada, 1992]. Fur- 

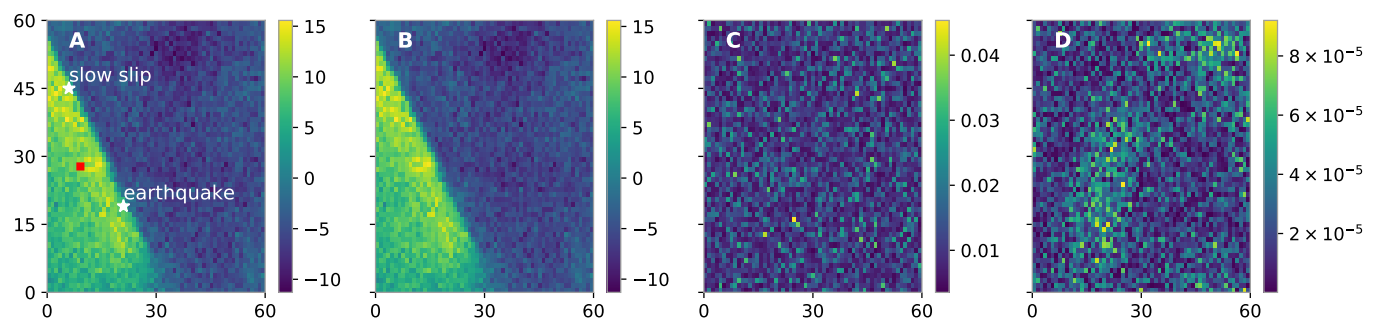

Figure 2. Performance of the Kalman filter (KF) for a two-dimensional synthetic deformation field. The phase evolution with its noise content is retrieved from noisy interferograms (i.e. non-zero network misclosure). $A$ : True cumulative phase at the last time-step of the time series including deformation and correlated and uncorrelated noise. $B$ : Reconstructed cumulative phase from the KF. $C$ : Root Mean Square error (RMS) of the the retrieved phase $(B)$ with respect to the true phase value $(A) . D$ : RMS of the phase retrieved with the $\mathrm{KF}(B)$ with respect to the least-squares solution. All scales are in centimeters to ensure the example represents a realistic case study. The location of the pixel of interest for Figure 3 is marked by a red square.

thermore, we include a constant deformation rate related with interseismic loading on the main fault and seasonal oscillations (i.e. yearly sinusoidal deformation with a phase shift) of the ground everywhere. In the following tests, we aim to recover all terms of deformation described above as well as the resulting phase evolution with our KF.

Consequently, the chosen parametrized model of the phase, $\phi_{k}$, at a time $t_{k}$, is

$$
\phi_{k}=a_{0}+a_{1} t_{k}+a_{2} \sin \left(t_{k} \frac{2 \pi}{T_{\text {year }}}\right)+a_{3} \cos \left(t_{k} \frac{2 \pi}{T_{\text {year }}}\right)+a_{4} S_{\text {sse }}\left(t_{k}\right)+a_{5} H_{\text {eq }}\left(t_{k}\right)+\gamma_{k},
$$

where $a_{n}, \forall n \in[1,5]$, are the parameters to be solved for, $T_{\text {year }}$ is a one year period, $S_{s s e}$ is an integrated spline function centered on day 210 with a width of $100, H_{e q}$ is a Heaviside function on day 500 and $\gamma_{k}$ is the mismodeling term at time $t_{k}$ with standard deviation $\sigma_{\gamma}$.

We first test the performance of the filter on synthetic data without any noise (implying $\left.\gamma_{k}=0, \forall k\right)$ and then on data including noise. To do so, we design a composite noise model to mimic real observations. This implies that we have, first, a spatially correlated noise reflecting atmospheric effects on each phase 'acquisition' and, secondly, a misclosure error lower by at least an order of magnitude, assuming that we have no contribution from unwrapping errors [Schmidt \& Bürgmann, 2003, Lohman \& Simons, 2005, Cav- 
alié et al., 2007, López-Quiroz et al., 2009, Agram \& Simons, 2015]. We add spatial and temporal deviations to all parameters $a_{n}$ following a random distribution with a standard deviation equal to $10 \%$ of their values, and random noise on interferogram construction equivalent to $\sigma_{\epsilon}=0.1 \mathrm{~mm}$. Moreover, the atmospheric contribution to phase decorrelation is constructed through the convolution of a white noise, with standard deviation of $10 \mathrm{~mm}$, and a decreasing exponential function of inter-pixel distances [Jolivet \& Simons, 2018]. The specified values reflect errors observed in processed Sentinel 1 data (see Section 3.3). The resulting cumulative phase change after 3 years is shown in Figure 2. The temporal evolution of phase change for one pixel is visible in Figure 3.

\subsubsection{Performance of the Kalman filter with optimal configuration}

We apply our KF with the assumption that the functional form given in Equation 6 is known. Results for simpler functional forms applied to the same synthetic data are in Figure S2 and S3. A priori model parameters in the initial state vector, $\mathbf{m}_{0}$, are set to zero with standard deviations comparable to the expected spread of parameters: $10 \mathrm{~mm}$ for $a_{0}, 0.05 \mathrm{~mm} /$ day for $a_{1}, 5 \mathrm{~mm}$ for the sine and cosine amplitudes and $70 \mathrm{~mm}$ for the displacement of slip events. The first phase value for all pixels is set to zero with zero uncertainty. This means that $\mathbf{m}_{0}$ is a null vector and $\mathbf{P}_{0}$ is a diagonal matrix containing the squared standard deviations listed above. When realistic noise is considered, we chose optimal parameters corresponding to the noise implemented in the synthetic data, that is $\sigma_{\gamma}=10 \mathrm{~mm}$ and $\sigma_{\epsilon}=0.1 \mathrm{~mm}$. For comparison, we solve the full problem for all acquisitions using an equivalent least squares inversion with identical model and data covariances [Tarantola, 2005].

For a model without any noise (except $\sigma_{\epsilon}=10^{-5} \mathrm{~mm}$ to avoid singularity of the gain), phase values are retrieved within $\sigma_{\epsilon}$ and model parameters converge after the assimilation of $\sim 6$ months of data (Figure 3A). The time required for convergence of the model parameters is justified by the fact that there is an ambiguity between the contribution of the linear and periodic terms to the deformation before reaching half the oscillation period. Regarding the earthquake, the corresponding amplitudes is found within $10^{-5}$ $\mathrm{mm}$ just after it occurred. Similarly, the amplitude of the slow slip event is retrieved once the total cumulative displacement caused by the slow slip event has been fully assimilated. 

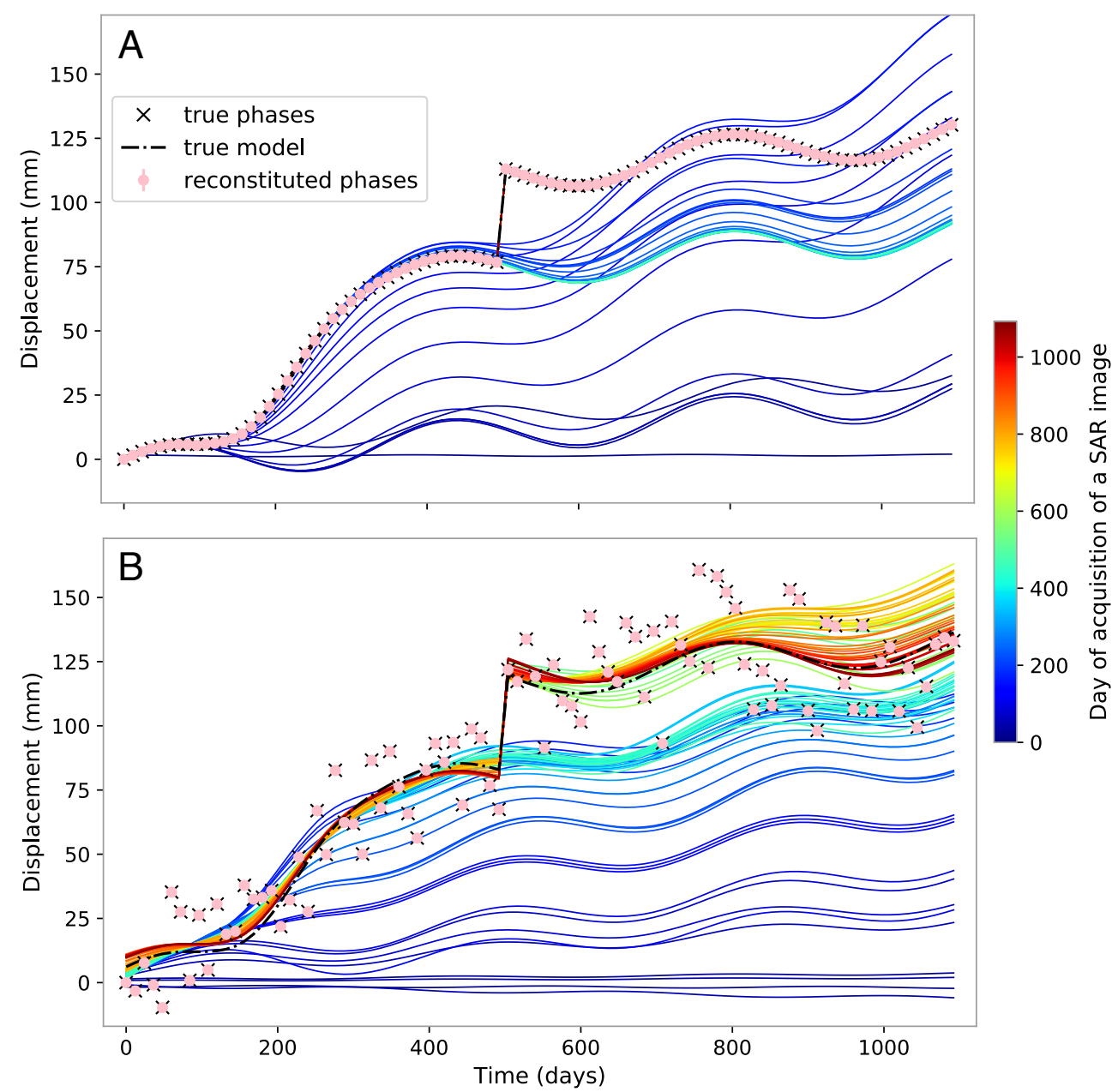

Figure 3. Time series for one pixel with temporal evolution of the model on a synthetic set of InSAR data for one pixel. $A$ : case without noise in the synthetic data. $B:$ same synthetic deformation but with a realistic noise model on top. Pink markers represent reconstituted phases from the Kalman filter, while black crosses are 'true' phases. When the phase is well retrieved, markers overlay each other and errorbars are too small to appear. Colored lines are models derived at each assimilation of a new acquisition, which date is indicated by the colorbar. Dashed black line is the true deformation. In $A$, true and reconstituted phases lie on each other and mask the underlying curves, which include the true model and computed models after day 500 (time of the modeled earthquake). 

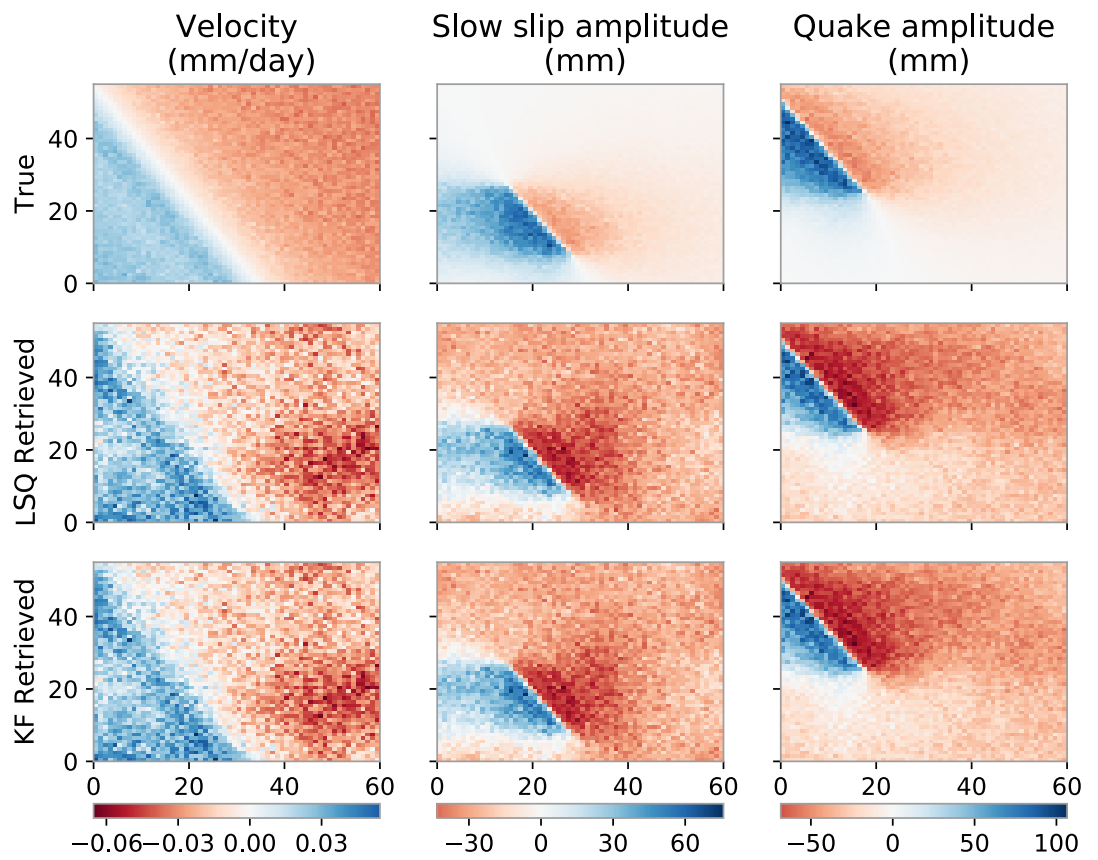

Figure 4. Maps of three of the model parameters : velocity, slow slip amplitude and quake amplitude. For comparison the true values (top), the values retrieved through least squares inversion (LSQ) (middle) and the values retrieved through Kalman filter (KF) (bottom) are displayed.

The final outcome of the KF is comparable with basic least squares performance (Figure 2 and 4 ). Figure 2 shows that the KF cumulative displacement root mean square error (RMS) with respect to the true displacement is on the order of $\sigma_{\epsilon}(0.1 \mathrm{~mm})$, while it is of $\sim 10^{-5} \mathrm{~cm}$ with respect to least squares estimation. Regarding model parameters, the difference between $\mathrm{KF}$ solution and target value is of $\sim 1 \mathrm{~mm}$, whereas it is of $\sim 10^{-3} \mathrm{~mm}$ between KF and least squares solutions. The noticeably large noise in retrieved parameters over areas with target values close to zero (Figure 4) is explained by the constant high a priori variance applied everywhere. Thus, if the location of the events is known, it is preferable to define a spatially variable a priori variance for, at least, slip events.

We detail here the behavior of the filter as data is assimilated in time and the requirements for convergence. Figure 3B shows the time series of a representative pixel (located on Figure 2) and Figure 5 the associated evolution of model parameters. The functional model evolves and gains information as data are successively assimilated. Graphically, the dark blue curves combine both the a priori null model and the little information brought 

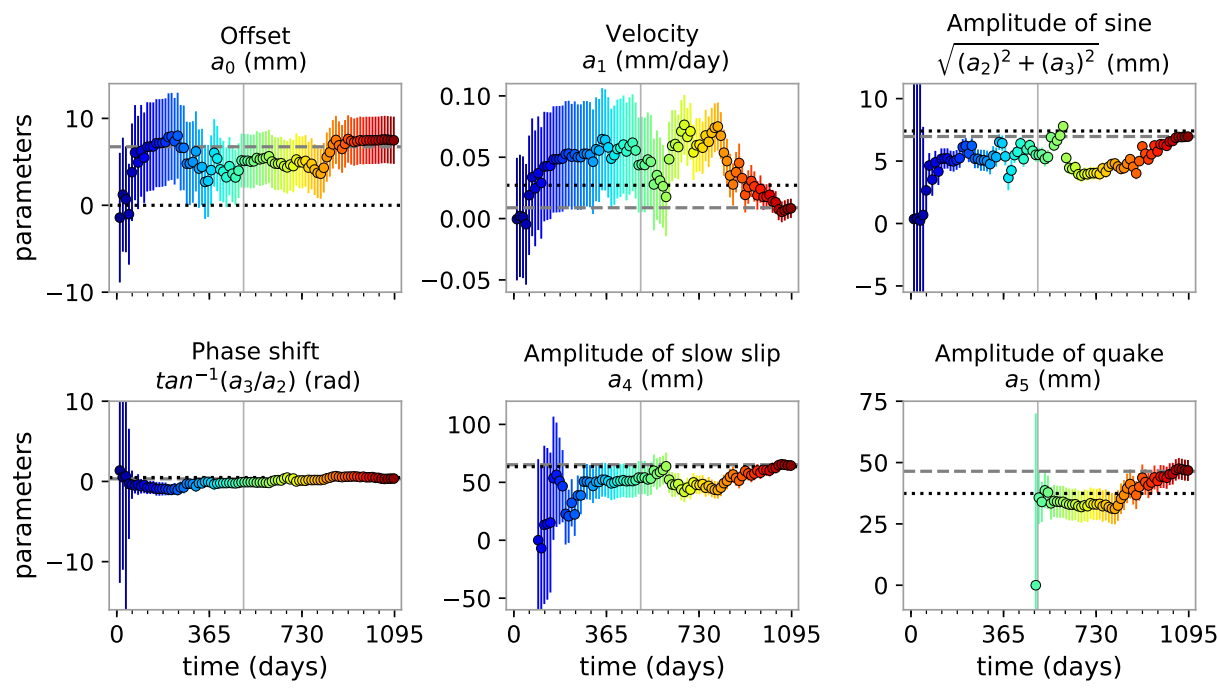

Figure 5. Temporal evolution of model functional parameters at each assimilation step for the reference pixel in our synthetic test. Colors refer to time (see colorbar in Figure 3). The amplitudes of the slow slip and the quake are added in the parameter space just before they occur. For reference, the dotted black line shows the true parameter value (i.e. target), and the dashed grey line shows the least-squares optimum. The Kalman filter solution tends toward the leastsquare solution, which itself depends on the interferometric network configuration and the noise in interferograms.

by the first few points, while the dark red curve uses all available information and closely fits the underlying model. The model progressively converges toward the least squares solution, close to the target model, at a rate that depends on how quickly parameter uncertainties collapse (Figures 3B and 5), which in turns depends on the Kalman gain (Equation 5, Appendix 7.1). As shown on Figure 5, it takes about 150 days for the offset, $a_{0}$, to be adjusted and around one year for the yearly periodic signal, $a_{2}$ and $a_{3}$. However, the inter-dependency of functional parameters clearly appears as variations in the transient event amplitude $a_{4}$ induces a change in $a_{0}$ by $1-2 \mathrm{~mm}$ and the earthquake at $t=$ 500 days perturbs almost all parameters, including the velocity which is shifted by $\sim 0.01$ $\mathrm{mm} /$ day. Correlations between parameters appear in the off-diagonal terms of the covariance matrix (Figure S1).

Interestingly, we also see that, although the local earthquake amplitude $a_{5}$ of $37.4 \mathrm{~mm}$ was correctly retrieved after 3 assimilations $\pm 7 \mathrm{~mm}$, the assimilation steps for $t>750$ days lead to an overestimate of $a_{5}$ and a correlated underestimate of $a_{1}$. As interfero- 

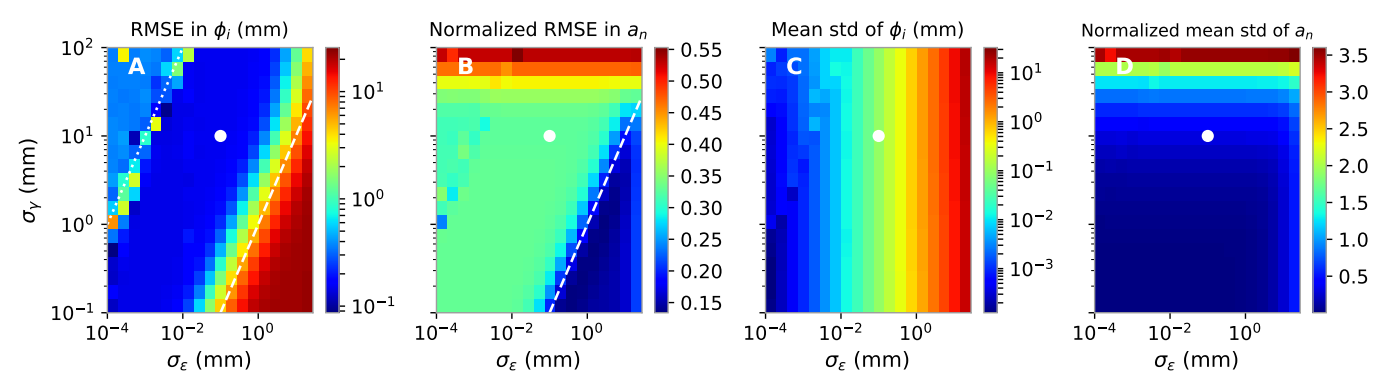

Figure 6. Errors in estimated model parameters $\left(a_{n}\right)$ and phases $\left(\phi_{i}\right)$ from the Kalman Filter $(\mathrm{KF})$ as a function of the standard deviation of the mismodeling noise $\left(\sigma_{\gamma}\right)$ and the standard deviation of interferometric network misclosure $\left(\sigma_{\epsilon}\right)$. The true value of the noise injected in the build of the synthetic deformation is marked by the white circle. $A$ and $B$ reveal variations in the KF estimate accuracy by looking at the Root Mean Square Error (RMSE) in $\phi_{i}(A)$ and in $a_{n}$ $(B) . C$ and $D$ display the mean standard deviation (abrv. std) of estimates. Values concerning parameters are normalized for homogeneity. The white dashed line corresponds to $\sigma_{\gamma} / \sigma_{\epsilon}=1$ and the dotted line to $\sigma_{\gamma} / \sigma_{\epsilon}=10^{4}$.

grams long after the event do not bring information about its amplitude, the state vector $\mathbf{m}_{k}$ needs to be modified. That is, to avoid fitting noise and limit trade off between parameters, functional parameters in $\mathbf{m}_{k}$ can be added or removed from the procedure when relevant. Also, phases which do not appear in latter interferograms can be stored and removed from $\mathbf{m}_{k}$. This does not affect final time series and lowers the memory load of the algorithm. Practically, our KF effectively works with two storage files : one containing time, phases and their uncertainties (from the diagonal of $\mathbf{P}_{k}$ ) and another one containing $\mathbf{m}_{k}, \mathbf{P}_{k}$ and other auxiliary information in order to run the next forecast and analysis at time $t_{k+1}$.

Nevertheless, it is a challenge to optimally parametrized the inversion with real InSAR data, especially because mismodeling and misclosure errors are generally not known[Schmidt \& Bürgmann, 2003].

\subsubsection{Sensitivity analysis to predefined errors}

We study the effect of non-representative $\sigma_{\gamma}$ and $\sigma_{\epsilon}$ in a sensitivity analysis, for one given pixel (shown in Figure 5 and 3). We deliberately set poorly chosen values of $\sigma_{\gamma}$ and $\sigma_{\epsilon}$ in the KF with respect to the known sources of noise. Subplots of Figure 6 display how 
4 proxies of the quality of KF estimates vary as a function of those two parameters. While Figures $6 \mathrm{~A}$ and $6 \mathrm{~B}$ consider the error in the absolute estimates, and thus the accuracy, Figures $6 \mathrm{C}$ and $6 \mathrm{D}$ indicate the precision (i.e. standard deviation) associated with those estimates.

The quantity displayed in $6 \mathrm{~A}$ is the Root Mean Square error of phase estimates, and in $6 \mathrm{C}$ it is the average of the posterior standard deviation of the phase estimates, $\sigma_{\phi_{k}}$. The exact equations corresponding to fields in $6 \mathrm{~B}$ and $6 \mathrm{D}$ are

$$
\sqrt{\frac{1}{L} \sum_{n=1}^{L}\left(\frac{a_{n}-a_{n}^{t}}{a_{n}^{t}}\right)^{2}} \text { and } \frac{1}{L} \sum_{n=1}^{L} \frac{\sigma_{a_{n}}}{\left|a_{n}\right|},
$$

respectively, where the superscript $t$ indicates target value and $L$ is the number of parameters. The first functional model parameters $a_{0}$, a constant offset, is voluntarily excluded because, first, $a_{n}^{t}=0$ and, second, the misfit in $a_{0}$ mainly results from the requirement that $\phi_{0}=0$.

As expected from governing equations, we see that estimated standard deviations are directly impacted by choices in $\sigma_{\gamma}$ and $\sigma_{\epsilon}$. Indeed, phase uncertainties appear sensitive to $\sigma_{\epsilon}$ and model parameter uncertainties to $\sigma_{\gamma}$ (Figure 6C, 6D). Another main feature is the improvement of $a_{n}$ estimates at the expense of the accuracy in $\phi_{k}$ when approaching the domain $\sigma_{\epsilon} \geq \sigma_{\gamma}$ (Figures $6 \mathrm{~A}, 6 \mathrm{~B}$ ). This is clearly not desirable, because phase estimate directly derive from interferometric data, while model parameters depend on an ad hoc functional description which may have to be improved as new data is assimilated. To the contrary, when too much confidence is given to interferograms with respect to the model (i.e. $\sigma_{\epsilon} \leq \sigma_{\gamma} \times 10^{-4}$ ) the effective misclosure error means that data may be hard to reconcile together and numerical instability arises. Thus, providing $\sigma_{\epsilon} / \sigma_{\gamma}$ is in between $10^{-4}$ and 1 , the quality of $\phi_{k}$ and $a_{n}$ estimates appears robust to several orders of magnitude variations in $\sigma_{\gamma}$ and $\sigma_{\epsilon}$. We still observe an upper bound limit of $\sim$ $30 \mathrm{~mm}$ and $\sim 15 \mathrm{~mm}$ for $\sigma_{\gamma}$ and $\sigma_{\epsilon}$, respectively, above which estimates are so uncertain that they do not adjust to the data. Time series representative of the overall effect of underestimating or overestimating $\sigma_{\gamma}$ and $\sigma_{\epsilon}$ are shown in supplementary Figures (S4S7).

In practice, $\epsilon_{i j}$ only results from how we construct interferograms and could be directly estimated by quantifying the effect of multilooking and filtering during the processing of each interferogram. Alternatively, it could be measured either before time series anal- 
ysis by forming triplets of interferograms [De Zan et al., 2015, Benoit et al., 2020] or $a$ posteriori by looking at the discrepancy between real and reconstituted interferograms from time series [Cavalié et al., 2007].

In addition to uncertainty quantification, another challenge of real InSAR data is that all interferograms do not unwrap everywhere due to local loss of coherence. Consequently, given pixels will potentially show missing links in the interferometric network. In the following we apply our KF to two data sets thought to be representative of the challenges brought by real InSAR data.

\subsection{Application to the Etna Volcano on Envisat Asar images}

As a real case example, we first present the assimilation of interferograms over the Etna volcano in Sicily. We have chosen this example as it has been used in the past for multiple validation studies [e.g. Doin et al., 2011, Jolivet et al., 2014] and because several GNSS stations record the relatively large displacements observed over this volcano. We use 63 images from the ENVISAT mission acquired between January 2003 and August 2010 [Doin et al., 2011]. Single Look Complex (SLC) images are focused and coregistered to a single master using the Repeat Orbit Inteferometry Package [ROIPac, Rosen et al., 2004]. Coregistration to a single master image is enhanced using the Digital Elevation Model and all possible interferograms are derived. The 222 interferograms are filtered and unwrapped using a branch cut algorithm [Goldstein et al., 1988, Goldstein \& Werner, 1998]. We correct interferometric phase delays due to the temporal variations of the stratified troposphere using the output of the ERA-Interim reanalysis of atmospheric data as described in Jolivet et al. [2011]. All details about the processing can be found in Doin et al. [2011] and in Jolivet et al. [2014].

We apply our Kalman filter (KF) on each pixel of the stack of images that has unwrapped interferometric data. In addition to the precise retrieval of phase evolution, we aim to obtain a mean rate of deformation, including potential transient events and ignoring seasonal contributions. Hence, our parametrized model for the phase evolution includes linear and seasonal terms described by four parameters, a constant term, a rate of phase change, a sine term and a cosine term. This model is very simplified for a volcano which has undergone several eruptive events over 2003-2010. Although this may lower our predictive capabilities, phase estimates of uninterrupted interferometric network should not 

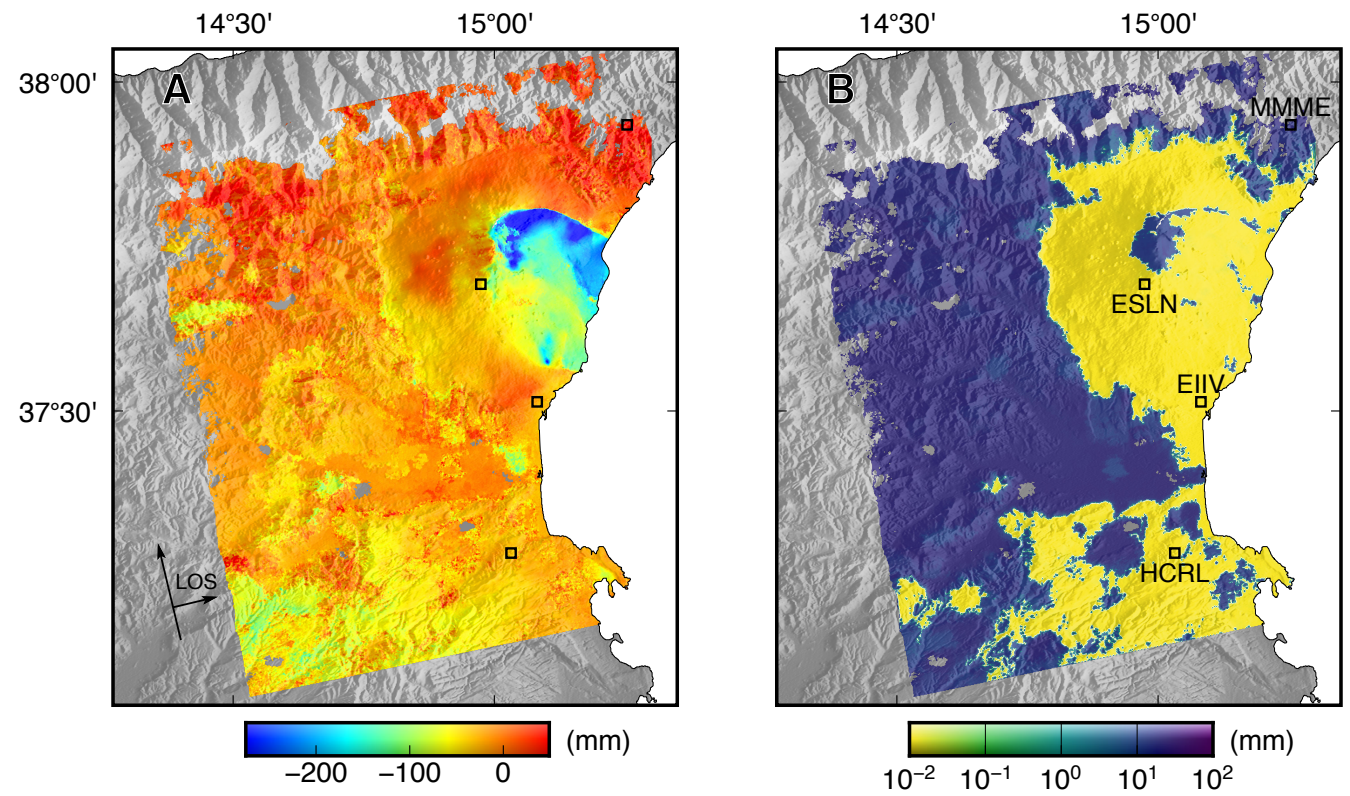

Figure 7. A: Map of cumulative phase change between 2003 and 2010 over Mt Etna (Sicily, Italy) as inferred from the Kalman filter time series analysis applied on ENVISAT data. B: Map of the associated standard deviation. Displacements and associated uncertainties are in the direction of the satellite's line of sight (LOS). Topography, shown in the background in shades of gray, is from SRTM [Farr et al., 2007]. Holes in the data correspond to pixels excluded from the inversion because less than 20 interferograms were unwrapped at their location. Black squares show a selection of GPS station used for comparison [Blewitt et al., 2018].

be affected and more complex model could be applied in a second time (Section 3.1). Consequently, we set $\sigma_{\gamma}=18 \mathrm{~mm}$ and $\sigma_{\epsilon}=0.01 \mathrm{~mm}$, as a high $\sigma_{\gamma}$ with respect to $\sigma_{\epsilon}$ limits the confidence in the model-based phase forecast and keeps large uncertainties for model parameters. With the same logic, we chose a priori standard deviation equal to $15 \mathrm{~mm}$ for the constant term, $5 \mathrm{~mm} /$ day for velocity and $10 \mathrm{~mm}$ for sine and cosine terms. We set the initial state vector $\mathbf{m}_{0}$ to zeros. Note that, because the constant term reflects the noise in the reference acquisition $\left(\phi_{0}\right)$ with respect to the model, its standard deviation should be close or superior to $\sigma_{\gamma}$. The impact of different $\sigma_{\gamma}$ and $\mathbf{P}_{0}$ on model parameter estimates is displayed in Figures S11-S13.

We compare local time series of displacement derived from Global Navigation Satellite System (GNSS), often referred to as GPS for simplicity, at the stations EIIV, ESLN, HCRL and MMME (Figures 7 and 8; Blewitt et al. [2018]). We consider differential displace- 
ments between two GPS stations and the equivalent closest InSAR pixel. Figure 7 shows the cumulative phase change and associated uncertainties over 8 years in the direction of the Line Of Sight (LOS) of the satellite as derived by our KF analysis at $t_{61}$, the 14 th of July 2010. The displayed penultimate phase $\phi_{61}$ incorporates most of the studied deformation with limited uncertainty as it is a reanalyse phase, unlike the last phase.

Standard deviations in Figure 7B are marked by a clear spatial dichotomy between the well resolved pixels displaying uncertainties $<10^{-1} \mathrm{~mm}$ and other pixels with uncertainties reaching more than $10 \mathrm{~mm}$. Precise estimates are available on the volcano flanks and in the urbanized region to the south, notably around stations ESLN, EIIV and HCRL and, thus, cover the area of geophysical interest. In Figure 7A, the displacement field is dominated by aseismic slip along the Pernicana fault extending from the volcano summit to the eastern coast of Sicily [Palano et al., 2006]. Indeed, the fault slipped more than $25 \mathrm{~cm}$ locally in the LOS direction over the $\sim 8$ years covered by the time series. Smaller coherent displacements of a few $\mathrm{cm}$ on the volcano flanks are also recovered. In the plains surrounding Mt Etna, cm-scale uncertainties are associated with about $\pm 2 \mathrm{~cm}$ of sharp inter-pixel variations in the displacement field.

Large uncertainties arise in area where more than $50 \%$ of interferograms do not unwrap due to significant spatial noise (Figure S10). When a pixel is not unwrapped, no information is available at this location and the analysis step of the filter cannot be performed. In this configuration, the forecast made from the functional model is taken as the estimate with its large uncertainty (i.e. $\mathbf{m}_{k}=\mathbf{m}_{k}^{f}$ and $\mathbf{P}_{k}=\mathbf{P}_{k}^{f}$ ). This allows to continue building the time series and to connect different subsets of interferograms which may not be linked by a common phase. However, the error associated with the forecast phase is carried forward in the subsequent solutions, if they are all relative to this one. A solution to lower uncertainties is to re-reference the phases by constructing long-baseline interferograms.

Time series in Figure 8 evidence that the relative InSAR displacement between pixels close to GPS stations is consistent with what is measured independently by GPS. A measure of the monotonicity of this relationship is given by the Spearman's rank correlation coefficient for $n$ pairs of InSAR-GPS observations, defined as

$$
C_{s}=1-\frac{6}{n\left(n^{2}-1\right)} \sum_{i=1}^{n} d_{i}^{2}
$$



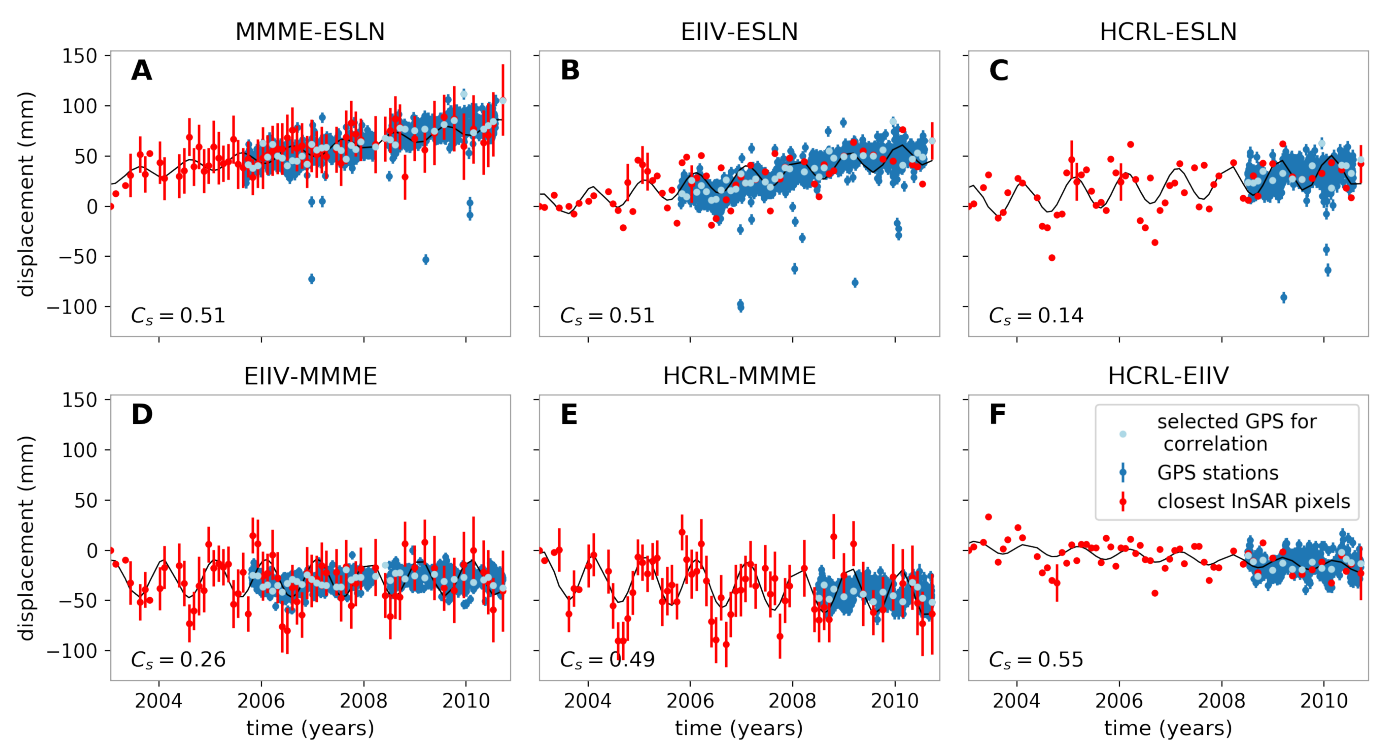

Figure 8. Overlay of GPS and InSAR time series of relative deformations. Each subplot includes the complete time series and its uncertainty for InSAR (red dots) and the available GPS time series on the same time period (blue dots). The subplot titles indicate the pair of stations located in Figure 7. The GPS data point that are temporally the closest to InSAR measurements are highlighted in light blue. They are used to compute the Spearman correlation coefficient, $C_{s}$ (Equation 8). The black line is the deformation according to the functional model of the Kalman filter on InSAR.

where $d_{i}$ is the difference between the ranks of the $i^{\text {th }}$ coeval observations in both sets. This metric was preferred over other correlation coefficients because of its little sensitivity to outliers. For the 6 differential displacements considered, $C_{s}$ is always positive indicating that when InSAR measures an increase, so does GPS (Figure 8). Moreover, its value close to 0.5 for 4 time series reveals a significant numerical correlation. Nonetheless, the implications of this metric are limited because it is applied to the subset of GPS measurement coincident in time with InSAR acquisitions and both time series are affected by different sources of noise. Independently of the numerical correlation, the overall good match between measured velocities validates our KF approach for InSAR time series analysis, even when the quality of data implies that errors are large (Figure 8A, $8 \mathrm{D}$ and $8 \mathrm{E})$. 


\subsection{Application to the Chaman fault on Sentinel 1 images}

In the following section, we illustrate the Kalman filter $(\mathrm{KF})$ performance to reconstruct surface displacements around a tectonic fault imaged by a recent satellite constellation. The satellites of the Sentinel 1 mission launched in 2014 and 2015, are providing SAR images with a better temporal sampling than ever before for C-band satellites. Over the Chaman fault, we construct 364 interferograms with 95 acquisitions spanning November 2014 to May 2019. We use the ISCE package (Gurrola et al. [2010]; JPL/Caltech) to build unwrapped interferograms. We coregister SAR images with a network-based enhanced spectral diversity (NESD) approach [Fattahi et al., 2017] and remove atmospheric perturbations using ECMWF-ERA 5 global reanalysis of atmospheric data (PyAPS software; Jolivet et al. [2011, 2014]). We also multilook and filter interferograms to enhance the signal-to-noise ratio before unwrapping with the branch cut method [Goldstein et al., 1988, Goldstein \& Werner, 1998]. The final size of the $2488 \times 7024$ pixels is $\sim 80 \times$ $130 \mathrm{~m}$. Additionally, we subtract to each interferogram a best fitting ramp (linear function of longitude and latitude) on the subregion north of the fault trace.

For comparison, we perform the time series analysis with both the well tested New Small Baseline Subset method, NSBAS [Berardino et al., 2002, Doin et al., 2011] implemented in GIAnT [Agram et al., 2013], and the KF developed here. We chose a simple functional description of deformation with a constant deformation rate and seasonal oscillations. A disadvantage of this model is that it poorly describes deformation for specific areas affected by earthquakes or anthropogenic groundwater pumping. However, because our implementation of NSBAS does not allow for spatial variations of the parametrized model, we prefer not to account for those very local dynamics (i.e. local in comparison with our $600 \mathrm{~km}$ long interferograms). Our a priori uncertainties are $25 \mathrm{~mm}$ for offset, $9 \mathrm{~mm} / \mathrm{yr}$ for velocity and $8 \mathrm{~mm}$ for cosine and sine terms. In agreement with Section 3.1.3, we chose $\sigma_{\gamma}=10 \mathrm{~mm}$ and $\sigma_{\epsilon}=0.05 \mathrm{~mm}$. Similarly to the previous example, we focus on the reconstructed phases with time rather than on its parametrized description and display maps of the penultimate phase of the time series (Figure 9).

Final solutions from NSBAS and KF are very similar (Figure 9A and 9B). The displacement relative to the first acquisition shows a long-wavelength fault-perpendicular gradient of about $60 \mathrm{~mm}$ over $\sim 120 \mathrm{~km}$. We also observe strong negative signals with a kilometer scale footprint, such as around the city of Quetta, most likely due to aquifer- 

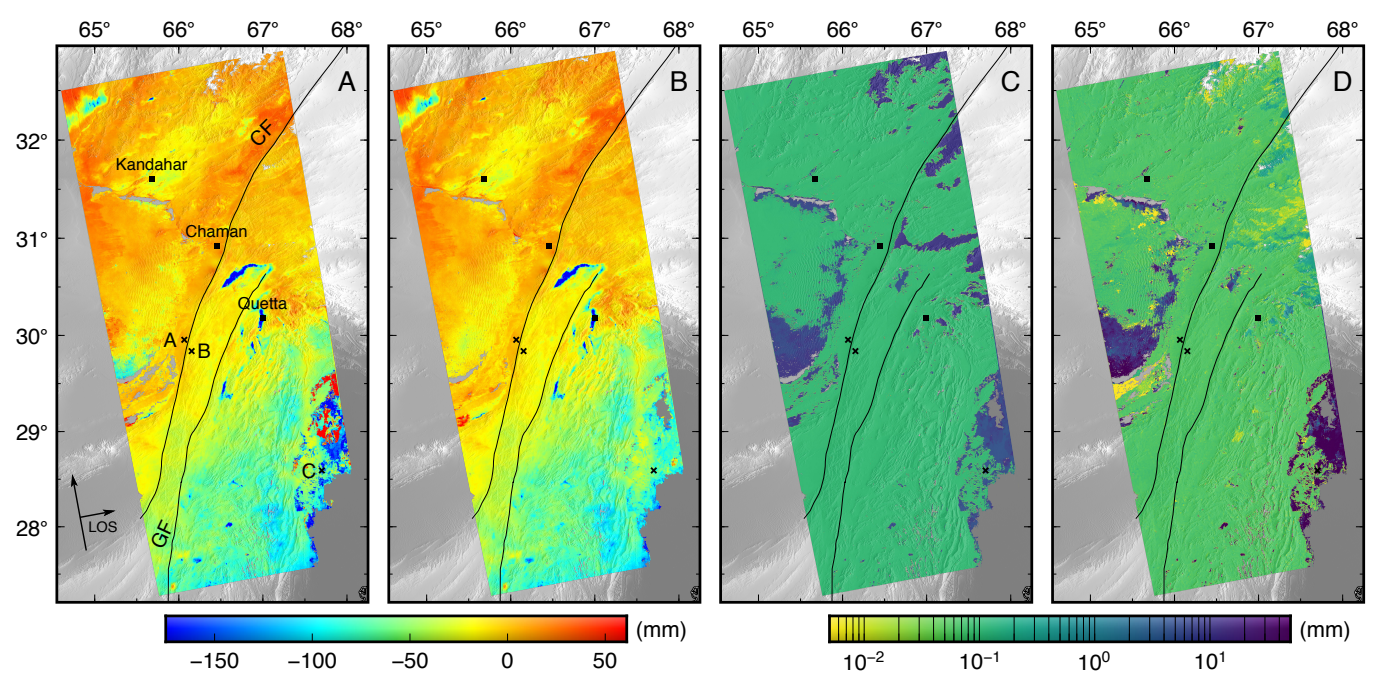

Figure 9. Maps of cumulative phase change between late 2014 and early 2019 over western Pakistan and southern Afghanistan from time series analyses applied on Track 42 data of Sentinel 1. A: Reference NSBAS solution, with labels of markers appearing in all subplots. $B$ and $C$ : Corresponding Kalman filter $(\mathrm{KF})$ solution with its standard deviation. $D$ : absolute difference between the NSBAS $(A)$ and $\operatorname{KF}(B)$ solutions. Displacements and associated uncertainties are in the direction of the satellite's line of sight. Two main faults of the region are the Chaman fault (CF) and the Ghazaband fault (GF) [Fattahi \& Amelung, 2016]. The topography is shown in the background in shades of gray. Cities are marked by square markers. The three black crosses and letters locate the selected pixels in subplots of Figure 10.

related subsidence. Moreover, there is a sharp contrast of displacement across the Chaman fault which reaches up to $\sim 3 \mathrm{~cm}$, notably in between labeled pixels A and B or across the northernmost segment, whereas no to little contrast is seen across the Ghazaband fault. This is consistent with Fattahi \& Amelung [2016] in depth study of the region. The difference in phase reconstruction between both methods is smaller than $0.1 \mathrm{~mm}$ after the assimilation of $\sim 4$ years of data, except in areas where the KF identified large uncertainty in the output with respect to the $\pm 0.05 \mathrm{~mm}$ precision (equal to $\sigma_{\epsilon}$ ) valid for most pixels (Figure 9C and 9D). Mismatch between NSBAS and KF methods, as well as large uncertainties in phase and model parameters concentrate around the dune desert to the West and the Indus River plain in the South-East corner. There, rapid geomorphological changes, seasonal oscillations and human activity impose a low interferometric coherence and, as a result, many interferograms could not be unwrapped there. 

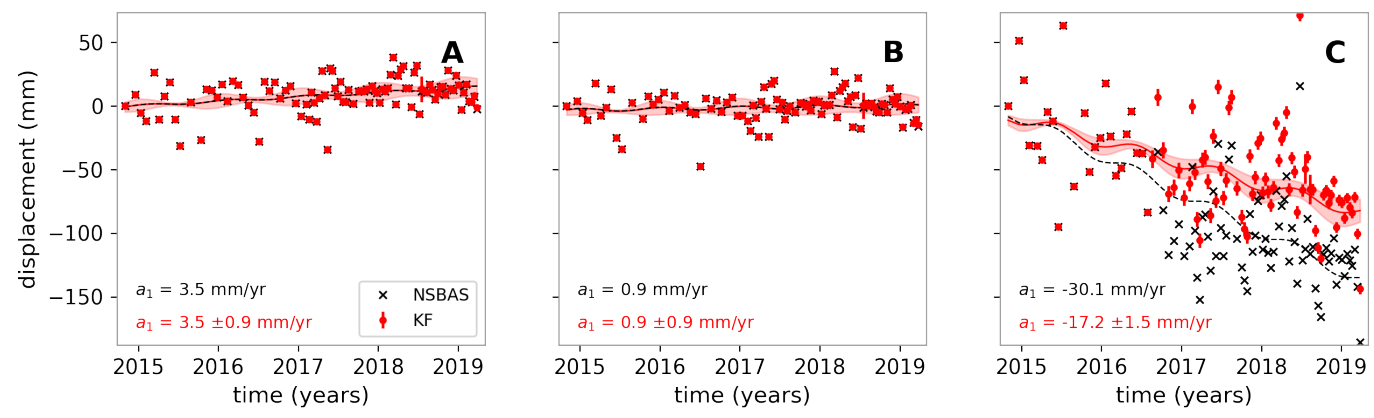

Figure 10. Time series for selected 3 pixels (indicated on Figure 9). Our Kalman filter (KF) solution with associated standard deviation (red dots) is shown alongside the solution computed with the NSBAS method [Berardino et al., 2002, Doin et al., 2011]. Most of the KF phase solutions have standard deviation too small to be visible. The corresponding functional models of deformation are also represented: dashed black curve for NSBAS and red line for KF. An idea of the spread of the models within one standard deviation of the KF solution is outlined by the red shaded area delimited by the parametrized phase evolution given $a_{i} \pm \sigma_{a_{i}}$ (Equation 2).

The concordance is also true at all time steps for every parameter of the state vector (displacements and functional model). Figure 10 presents three representative time series of deformation on single pixels. The two nearby pixels selected in Figure 10A and 10B exhibit highly correlated deformation with a spread of $\sim 7 \mathrm{~cm}$ around the functional model. Phase estimation is precise with $\pm 0.05 \mathrm{~mm}$ and in good agreement with NSBAS estimations. For pixel A, the inferred velocity is found to be $3.5 \pm 0.9 \mathrm{~mm} / \mathrm{yr}$ in the KF or 3.5 in the NSBAS solution and, similarly, the seasonal amplitude and phase shift are $0.9 \pm 1.2 \mathrm{~mm}$ and $0.7 \pm 0.1 \mathrm{rad}$ or $0.9 \mathrm{~mm}$ and $0.74 \mathrm{rad}$. Those functional descriptions agree with each other and the uncertainties given by the KF are precious indicators of the model representativity and, thus, of the confidence in the resulting forecast.

The time series in Figure 10C exhibits large error bars of $\sim 5 \mathrm{~mm}$ from mid-2016 arising from disconnected subsets in the interferogram network. In the KF, the use of the functional model to forecast a disconnected phase and link subnetworks means that the model error propagates to subsequent phase estimations. The parametrized model of deformation differs sensibly between NSBAS and KF methods (Figure 10), by opposition to the very good agreement found between KF and least-squares for synthetic data (Figure 4). A first reason for this is the a priori on model parameters in the KF, which effectively is a form of regularization leading to smaller velocity estimates of $a_{1}=-19$ 
$\mathrm{mm} / \mathrm{yr}$ instead of the physically unlikely $-40 \mathrm{~mm} / \mathrm{yr}$ for NSBAS. A second reason is that the NSBAS solution does not account for errors in model and data directly and instead attributes a weight to phase fitting over model adjustment, so that Equation 2 only impacts phase values when interferometric connections do not allow an estimate with Equation 1 [Doin et al., 2011] . By definition, this weight is similar to the ratio $\sigma_{\epsilon} / \sigma_{\gamma}$, and thus was set to $10^{-3}$.

Therefore, our Kalman filter can be applied to old, often sparse and incomplete data (ENVISAT) as well as to most recent and memory-consuming data which are collected nowadays (Sentinel 1).

\section{Discussion}

\subsection{Guideline to choose parameters}

Efficient KF requires a sensible parametrization of the problem, with the definition of appropriate errors $\left(\sigma_{\epsilon}, \sigma_{\gamma}\right)$, descriptive functional model $\left(f_{n}\left(t_{k}\right), \forall n\right)$ and a priori knowledge on the model parameters in $\mathbf{m}_{0}$ and $\mathbf{P}_{0}$ (Section 2.3).

For the functional model, it is a good rule of thumb to start with a simple model, which includes a linear polynomial and an annual periodic oscillation. The innovation or residual term is key to assess the relevance of this parametrized model, as it quantifies the difference between the data and the information brought by the forecast (Section 2.2). If the model is appropriate the mean innovation at each time step should have a Gaussian distribution around zero on each pixel (e.g. subplots B,C versus D,E in Figure S3). Thus, during the iterative process, checking the distribution of this mean innovation would help refine the parametrized model. Moreover, some source of deformation are a priori known and can be included. For instance, the displacements due to earthquakes affecting the study area can be easily included using a step function, which footprint is constrained by the location and timing of the event in seismic catalogs. To adapt to the diversity of applications of our KF, other implemented functions include higher degree polynomials, hyperbolic tangent, exponential and logarithmic decay as well as basis spline.

Further assumptions are required on the functional model when evaluating the a priori state $\mathbf{m}_{0}$ and covariance $\mathbf{P}_{0} \cdot \mathbf{m}_{0}$ and $\mathbf{P}_{0}$ define expected values of the multiplicative coefficients $a_{n}$ to each functional element $f_{n}$. Assuming $a_{n}$ terms are unknown, $\mathbf{m}_{0}$ is set to zero vector with a likely spread in the diagonal of $\mathbf{P}_{0}$. Consequently, small $\mathbf{P}_{0}$ dims 

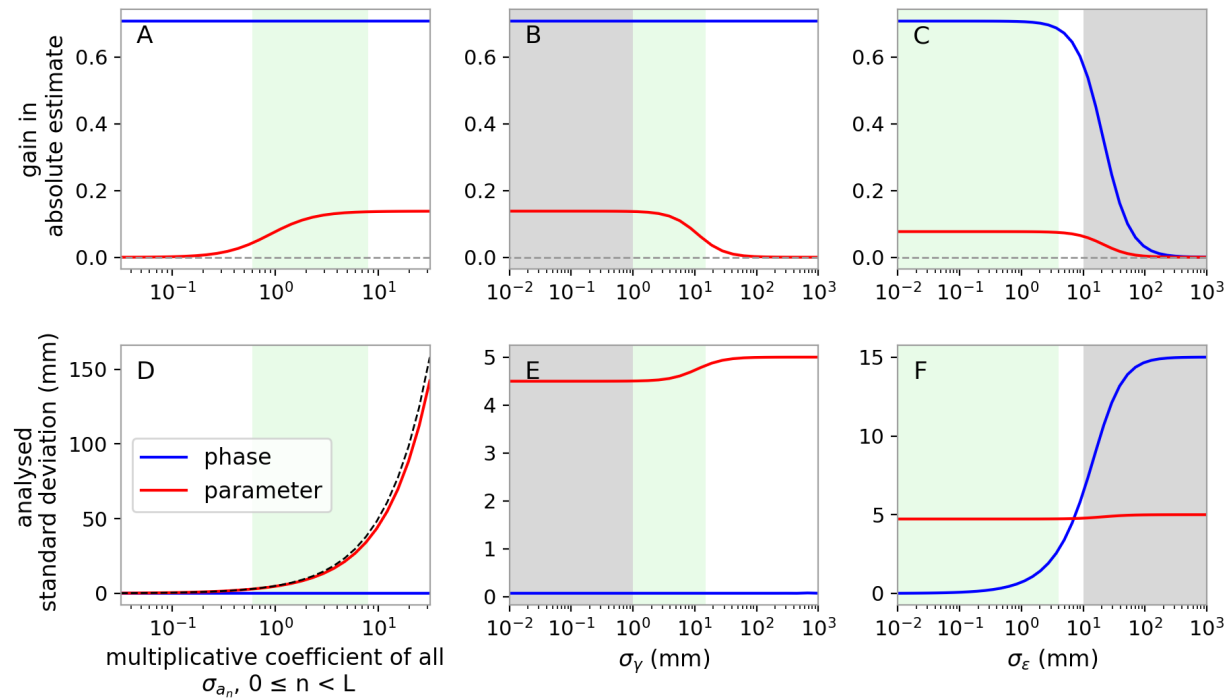

Figure 11. Variations of the gain in phase and model parameter estimates $(A-C)$ and of the standard deviation of those estimates $(D-F)$ as a function of the error terms used to initialize our Kalman Filter. Quantitative values are derived from Equations 3, 4 and 5 for the parametrization chose in our synthetic example. We look at one assimilation step $k=1$. The blue line is effectively $\phi_{1}$ and the red line $a_{3}$. Similar trends, with different amplitudes are observed for any $a_{n}$. We vary successively all $\sigma_{a_{n}}$ (in the diagonal of $\left.\mathbf{P}_{0}\right)(A, D), \sigma_{\gamma}(B, E)$ and $\sigma_{\epsilon}(C, F)$. In $A, D \sigma_{a_{n}}$ are multiplied by a common coefficient. The dashed grey line indicates zero gain. The black dashed line correspond to the case where $\sigma_{a_{3}}$ evaluated at $k=1$ is the a priori $\sigma_{a_{3}}$ (at $k=0$ ). The shaded grey area is the domain where $\sigma_{\epsilon} \dot{i} \sigma_{\gamma}$. Green shading highlights the domains where the (i) gain on the phase is maximal, (ii) the gain on model parameter is not null (i.e. the model learns from data), (iii) the uncertainty on phase estimate is small $\left(\sim 10^{-2} \mathrm{~mm}\right)$ and (iv) the uncertainty on parameter estimate is not very large $(<40 \mathrm{~mm})$. Variations in $\sigma_{\gamma}$ or $\sigma_{a_{n}}$ do not affect phase estimates. The model parameters are not adjusting to the data when the a priori on its standard deviation is too low or when $\sigma_{\gamma}$ is too high.

extrema as it is effectively a regularization term for $a_{n}$, while large $\mathbf{P}_{0}$ allows parameters to adjust freely to incoming data and stability might be lost (Figure 11, S8, S9 and S12). In practice, the order of magnitude of a priori errors is determined using our physical knowledge about expected deformation. Higher values will be favored if little smoothing of the model is desired, however, this may lead to unrealistic forecast and very large 
$\sigma_{a_{n}}$ in the first few assimilation steps. The impact of the $\mathbf{m}_{0}$ and $\mathbf{P}_{0}$ will tend to vanish as more data is assimilated.

Regarding the mismodeling noise $\gamma_{k}$ and network misclosure $\epsilon_{i j}$ (Equations 1,2), we assumed that they could be represented by constant standard deviations $\sigma_{\gamma}$ and $\sigma_{\epsilon}$, unless variations in acquisition quality (e.g. seasonal noise from snow cover) or in interferogram construction (e.g. varying amount of filtering) are known. Typically, we have $\sigma_{\epsilon} \ll$ $\sigma_{\gamma}$ so that phase fitting is strongly favored over parametrized model adjustment (Section 3.1.3, Figure 11). $\epsilon_{i j}$ could be measured by looking at the closure of triplets of interferograms and representative $\sigma_{\epsilon}$ deduced. $\sigma_{\gamma}$ should reflect the dispersion of the data around the parametrized model, which depends on the chosen model itself and the noise in the data. From previously published studies, $\sigma_{\epsilon}$ is of the order of the millimeter (assuming no unwrapping error) and $\sigma_{\gamma}$ superior to the centimeter [Schmidt \& Bürgmann, 2003, Cavalié et al., 2007, López-Quiroz et al., 2009, Sudhaus \& Jónsson, 2009, Agram \& Simons, 2015].

\subsection{Efficiency of the Kalman filter}

A main improvement of the Kalman Filter (KF) over more conventional method is the data assimilation approach. We have shown that it is capable of accurately solving the same problem than a least-squares method. However, our Kalman Filter is designed to solve other problems relevant to our ever-expanding SAR archive. First, it can actualize a pre-existing time-series with new interferograms in a fast and neat way. Secondly, we have built the tool in a modular and flexible manner, so that it can adapt to the evolving knowledge of the deformation as data is assimilated. Below, we discuss and detail those statements.

The iterative procedure allows fundamental discussion about the amount and shape of data necessary to obtain a meaningful description of deformation. Our tests reveal that phases are instantaneously fitted to $\pm 0.1 \mathrm{~mm}$ with later refinement as we gain information from new interferograms. Differently, model parameters require at least one year of data in order to converge, a time that depends on the variability of deformation measured and how precise and accurate is our a priori knowledge. Velocity adjusts rather quickly, if no transient event is recorded, compared to the cosine and sine terms which require obviously more than a year. Consequently, forecast within the first year is rarely 
accurate, which clearly reflects on the uncertainty. Once model parameters have converged toward their final value, the forecast is as good as the model is, independently of the elapsed time of assimilation. The instantaneous innovation reflects the dispersion of the data around the background model.

Updating pre-existing model imply a gain of time, computing power and memory. Quantitative comparisons of computing performance of NSBAS and KF methods are not easy because their implementation are different. Indeed, our KF is implemented for simultaneous processing of pixels in parallel by Message Passing Interface, while NSBAS uses multiple threads with shared memory in its GIAnT version [Agram et al., 2013]. However, it is clear that the numerical cost of updating an existing time series with the KF is much smaller than when retrieving all the phases at once with NSBAS. The time to run a KF update incorporates not only the computation time, but also the time necessary to read and write data and models. The latest has been optimized so that, in the example presented in Section 3.2, reconstructing phases and parameters for the 62 first dates takes $17 \mathrm{~min}$, whereas updating the time series with the last acquisition takes only $30 \mathrm{sec}$. As a reference, we use 2 computing nodes with 20 threads per nodes and InfiniBand communication. Concerning memory usage, previously computed interferograms do not need to be stored in order to update existing model, providing that the latest estimates of $\mathbf{m}_{k}$ and $\mathbf{P}_{k}$ are available. For the example in Section 3.3, this information is stored in a HDF5 file of 6 Go, while all interferograms weigh $>25$ Go.

Another advantage of the KF is the systematic and consistent propagation of error through time series analysis. It is a requirement to correctly combine what we know from the data and from the existing model. We have seen that the absolute value of the uncertainty associated with computed phases is a consequence of the a priori standard deviation of misclosure $\left(\sigma_{\epsilon}\right)$ (Figure 6), which can be measured from interferograms or inferred from the way interferograms are built. Additionally, the standard deviation of mismodeling error $\left(\sigma_{\gamma}\right)$ will also come into play in the case of missing data for a time step or disconnection in the interferometric network. This error is a more subjective parameter, as it depends on the functional description chosen, and the dispersion of phases around it. Nevertheless, the relative uncertainty in between pixels and time steps directly results from the data structure, such as the number of interferograms available or how 'far' is the temporal reference. Those differences allow us to discriminate pixels and weight estimates for subsequent processing or modeling. This is particularly relevant for long time-series 
( $<1$ year). Furthermore, covariance estimation is key to combine different data sets, such as InSAR and GPS [Bekaert et al., 2016, Sudhaus \& Jónsson, 2009] or different frames of InSAR acquisitions [Jolivet \& Simons, 2018].

\subsection{Limitation and perspectives}

The propagation of uncertainty highlights a fundamental limit of time series analysis. The time series being relative to the first date, errors tend to propagate in time. This is a big issue for long time series, such as ones drawn from Sentinel 1 nowadays. Numerous interferograms, especially those with long baselines, are necessary to limit this effect inherent to any classical InSAR time series analysis. Moreover, the similarity with GPS time series evidenced in Section 3.2 shows the possibility of a precise re-referencing of the pixel location within the time series. This has been done for vertical displacement [Shirzaei \& Bürgmann, 2018]. In addition, our comparison with GPS time series could be improved by correcting interferograms for ionospheric effect [Simons \& Rosen, 2015, Liang et al., 2019].

The pixel by pixel approach of our KF imply that we do not account for spatial covariance [Jolivet \& Simons, 2018]. This covariance may take the form of a function of the pixel-to-pixel distance, which empirically models the isotropic part of the InSAR signal not due to ground deformation. Such signal mainly arises from atmospheric effects. In our real case example, we limited the spatial correlation by substracting a best-fitting ramp to interferograms and by removing the stratified tropospheric delays in each interferogram. Turbulent atmospheric delays remain, however. Nevertheless, because our $\mathrm{KF}$ is built to deal with long time series, the temporally decorrelated contributions of InSAR (e.g. turbulent delays) are reflected by the inter-acquisition dispersion for a given pixel and is empirically included in the mismodeling error. This contrast with studies looking at few SAR acquisitions to deal with a localized event in time [Lohman \& Simons, 2005, Sudhaus \& Jónsson, 2009]. Spatial covariances are also implemented to increase spatial continuity [Jolivet \& Simons, 2018]. For our KF, we found that spatial continuity of phase and function parameter naturally arise from the data which only has high inter-pixel noise in regions where coherence is low (e.g. Figure 10). In such region, the numerous 'holes' in assimilated interferograms ensure low confidence in the KF estimates. Spatial constrain would help gain confidence by adding more information in the problem but it would dramatically increase the numerical cost and would require additional 
parametrization [Agram \& Simons, 2015, Jolivet \& Simons, 2018]. Additionally, the smoothing of model parameters brought by the a priori ensure greater spatial continuity in low coherence area with respect to NSBAS.

We built the Kalman filter as an accessible tool relevant to many geophysical applications. More specific applications will be implemented in the future, taking advantage of the iterative procedure as well as systematically exploiting outputs of the KF not detailed in this paper, such ad the full temporal covariance matrix or the gain and innovation vectors. For instance, the iterative procedure is ideal to implement automatic detection of transient events, such as slow slip on faults. The quality of the parametrized model could be systematically checked by looking at the instantaneous innovation of phase values but also of model parameters. An automatic detection of non-gaussianity of the innovation distribution over time could send a warning, stop the assimilation and or automatically update the model with predefined functions (e.g. quadratic term, Heaviside function). Another major improvement of our KF would be to remove $\sigma_{\gamma}$ from the predefined parameters and include it as a parameter to be recovered during time series analysis.

\section{Conclusion}

We developed a tool to rapidly and efficiently update pre-existing time series of deformation from a set of unwrapped interferograms as they are made available. The Kalman filter (KF) approach is new to InSAR time series analysis and was tested on diverse sets of synthetic and real interferograms in regions affected by tectonic deformations. We show that the filter behaves in agreement with existing methods and GNSS measurements, providing that we correctly estimate errors associated with interferograms as well as with the parametrized description of deformation. We thoroughly studied and described the design and impact of setup parameters. The source code is fully implemented in Python 3 and was built as a flexible and modular tool for the community.

\section{Acknowledgments}

This article benefited from previous works by Angelique Benoit and discussions with Dr Marie Bocher, Pr Alexandre Fournier, Dr Kristel Chanard, Pr Emmanuel Cosme and Theo Rebert. This work received funding from the European Research Council (ERC) under the European Union's Horizon 2020 research and innovation program (Geo-4D project, 
grant agreement 758210). Data sets used in this study are freely available online. Synthetic Aperture Radar images are from the PEPS platform (Sentinel 1) and from ESA Earth Online (Envisat). The digital elevation model is from NASA EarthData. ERA5 global reanalyses of atmospheric data are distributed by the ECMWF. GPS time series come from the Nevada Geodetic Laboratory website. The source code is available on Github at https://github.com/ManonDls/KFTS-InSAR.

\section{References}

Agram, P., Jolivet, R., Riel, B., Lin, Y., Simons, M., Hetland, E., ... Lasserre, C. (2013). New radar interferometric time series analysis toolbox released. Eos, Transactions American Geophysical Union, 94(7), 69-70.

Agram, P., \& Simons, M. (2015). A noise model for insar time series. Journal of Geophysical Research: Solid Earth, 120(4), 2752-2771.

Bato, M. G., Pinel, V., Yan, Y., Jouanne, F., \& Vandemeulebrouck, J. (2018). Possible deep connection between volcanic systems evidenced by sequential assimilation of geodetic data. Scientific reports, 8(1), 1-13.

Bekaert, D., Segall, P., Wright, T. J., \& Hooper, A. J. (2016). A network inversion filter combining gnss and insar for tectonic slip modeling. Journal of Geophysical Research: Solid Earth, 121(3), 2069-2086.

Benoit, A., Pinel-Puysségur, B., Jolivet, R., \& Lasserre, C. $\quad$ (2020, 03). Corphu: an algorithm based on phase closure for the correction of unwrapping errors in sar interferometry. Geophysical Journal International, 221(3), 1959-1970. doi: $10.1093 /$ gji/ggaa120

Berardino, P., Fornaro, G., Lanari, R., \& Sansosti, E. (2002). A new algorithm for surface deformation monitoring based on small baseline differential sar interferograms. IEEE Transactions on Geoscience and Remote Sensing, 40(11), $2375-2383$.

Biggs, J., Ebmeier, S., Aspinall, W., Lu, Z., Pritchard, M., Sparks, R., \& Mather, T. (2014). Global link between deformation and volcanic eruption quantified by satellite imagery. Nature communications, 5, 3471.

Blewitt, G., Hammond, W., \& Kreemer, C. (2018). Harnessing the gps data explosion for interdisciplinary science. Eos, 99 .

Burgmann, R., Rosen, P. A., \& Fielding, E. J. (2000). Synthetic aperture radar in- 
terferometry to measure earth's surface topography and its deformation. Annual review of earth and planetary sciences, 28(1), 169-209.

Carrassi, A., Bocquet, M., Bertino, L., \& Evensen, G. (2018). Data assimilation in the geosciences: An overview of methods, issues, and perspectives. Wiley Interdisciplinary Reviews: Climate Change, 9(5), e535.

Cavalié, O., Doin, M.-P., Lasserre, C., \& Briole, P. (2007). Ground motion measurement in the lake mead area, nevada, by differential synthetic aperture radar interferometry time series analysis: Probing the lithosphere rheological structure. Journal of Geophysical Research: Solid Earth, 112(B3).

Chaussard, E., Bürgmann, R., Shirzaei, M., Fielding, E., \& Baker, B. (2014). Predictability of hydraulic head changes and characterization of aquifer-system and fault properties from insar-derived ground deformation. Journal of Geophysical Research: Solid Earth, 119(8), 6572-6590.

Chaussard, E., Wdowinski, S., Cabral-Cano, E., \& Amelung, F. (2014). Land subsidence in central mexico detected by alos insar time-series. Remote sensing of environment, 140, 94-106.

Cohn, S. E. (1997). An introduction to estimation theory (gtspecial issueltdata assimilation in meteology and oceanography: Theory and practice). Journal of the Meteorological Society of Japan. Ser. II, 75(1B), 257-288.

Cohn, S. E., Sivakumaran, N., \& Todling, R. (1994). A fixed-lag kalman smoother for retrospective data assimilation. $\quad$ Monthly Weather Review, 122(12), 28382867.

Cosme, E., Verron, J., Brasseur, P., Blum, J., \& Auroux, D. ～(2012). Smoothing problems in a bayesian framework and their linear gaussian solutions. Monthly Weather Review, 140(2), 683-695.

De Zan, F., Zonno, M., \& López-Dekker, P. (2015). Phase inconsistencies and multiple scattering in sar interferometry. IEEE Transactions on Geoscience and Remote Sensing, 53(12), 6608-6616.

Doin, M.-P., Guillaso, S., Jolivet, R., Lasserre, C., Lodge, F., Ducret, G., \& Grandin, R. (2011). Presentation of the small baseline nsbas processing chain on a case example: The etna deformation monitoring from 2003 to 2010 using envisat data. In Proceedings of the fringe symposium (pp. 3434-3437).

Elliott, J., Walters, R., \& Wright, T. (2016). The role of space-based observation 
in understanding and responding to active tectonics and earthquakes. Nature communications, 7, 13844.

Emardson, T., Simons, M., \& Webb, F. (2003). Neutral atmospheric delay in interferometric synthetic aperture radar applications: Statistical description and mitigation. Journal of Geophysical Research: Solid Earth, 108(B5).

Evensen, G. (2009). Data assimilation: the ensemble kalman filter. Springer Science \& Business Media.

Farr, T. G., Rosen, P. A., Caro, E., Crippen, R., Duren, R., Hensley, S., ... others (2007). The shuttle radar topography mission. Reviews of geophysics, 45(2).

Fattahi, H., Agram, P., \& Simons, M. (2017). A network-based enhanced spectral diversity approach for tops time-series analysis. IEEE Transactions on Geoscience and Remote Sensing, 55(2), 777-786.

Fattahi, H., \& Amelung, A. (2016). Insar observations of strain accumulation and fault creep along the chaman fault system, pakistan and afghanistan. Geophys. Res. Lett., 43, 8399-8406. doi: 10.1002/2016GL070121

Ferretti, A., Prati, C., \& Rocca, F. (2000). Nonlinear subsidence rate estimation using permanent scatterers in differential sar interferometry. IEEE Transactions on geoscience and remote sensing, 38(5), 2202-2212.

Goldstein, R. M., \& Werner, C. L. (1998). Radar interferogram filtering for geophysical applications. Geophysical research letters, 25(21), 4035-4038.

Goldstein, R. M., Zebker, H. A., \& Werner, C. L. (1988). Satellite radar interferometry: Two-dimensional phase unwrapping. Radio science, 23(4), 713-720.

Grandin, R., Doin, M.-P., Bollinger, L., Pinel-Puysségur, B., Ducret, G., Jolivet, R., \& Sapkota, S. N. (2012). $\quad$ Long-term growth of the himalaya inferred from interseismic insar measurement. Geology, 40(12), 1059-1062.

Griffiths, H. (1995). Interferometric synthetic aperture radar. Electronics \& communication engineering journal, 7(6), 247-256.

Gurrola, E., Rosen, P., Sacco, G., Seliga, W., Zebker, H., Simons, M., \& Sandwell, D. (2010). Insar scientific computing environment. In 2010 american geophysical union meeting.

Hetland, E., Musé, P., Simons, M., Lin, Y., Agram, P., \& DiCaprio, C. (2012). Multiscale insar time series (mints) analysis of surface deformation. Journal of Geophysical Research: Solid Earth, 117(B2). 
Hilley, G. E., Bürgmann, R., Ferretti, A., Novali, F., \& Rocca, F. (2004). Dynamics of slow-moving landslides from permanent scatterer analysis. Science, 304(5679), $1952-1955$.

Hofmann-Wellenhof, B., Lichtenegger, H., \& Collins, J. (2012). Global positioning system: theory and practice. Springer Science \& Business Media.

Hooper, A., Segall, P., \& Zebker, H. (2007). Persistent scatterer interferometric synthetic aperture radar for crustal deformation analysis, with application to volcán alcedo, galápagos. Journal of Geophysical Research: Solid Earth, 112(B7).

Jolivet, R., Agram, P. S., Lin, N. Y., Simons, M., Doin, M.-P., Peltzer, G., \& Li, Z. (2014). Improving insar geodesy using global atmospheric models. Journal of Geophysical Research: Solid Earth, 119(3), 2324-2341.

Jolivet, R., Grandin, R., Lasserre, C., Doin, M.-P., \& Peltzer, G. (2011). Systematic insar tropospheric phase delay corrections from global meteorological reanalysis data. Geophysical Research Letters, 38(17).

Jolivet, R., Lasserre, C., Doin, M.-P., Guillaso, S., Peltzer, G., Dailu, R., ... Xu, X. (2012). Shallow creep on the haiyuan fault (gansu, china) revealed by sar interferometry. Journal of Geophysical Research: Solid Earth, 117(B6).

Jolivet, R., Lasserre, C., Doin, M.-P., Peltzer, G., Avouac, J.-P., Sun, J., \& Dailu, R. (2013). Spatio-temporal evolution of aseismic slip along the haiyuan fault, china: Implications for fault frictional properties. Earth and Planetary Science Letters, 377, 23-33.

Jolivet, R., \& Simons, M. (2018). A multipixel time series analysis method accounting for ground motion, atmospheric noise, and orbital errors. Geophysical Research Letters, 45(4), 1814-1824.

Jolivet, R., Simons, M., Agram, P., Duputel, Z., \& Shen, Z.-K. ～(2015). Aseismic slip and seismogenic coupling along the central san andreas fault. Geophysical Research Letters, 42(2), 297-306.

Kalman, R. E. (1960). A new approach to linear filtering and prediction problems. Journal of basic Engineering, 82(1), 35-45.

Khoshmanesh, M., \& Shirzaei, M. (2018). Multiscale dynamics of aseismic slip on central san andreas fault. Geophysical Research Letters, 45(5), 2274-2282.

Liang, C., Agram, P., Simons, M., \& Fielding, E. J. (2019). Ionospheric correction of insar time series analysis of c-band sentinel-1 tops data. IEEE Transactions on 
Geoscience and Remote Sensing, 57(9), 6755-6773.

Lindsey, E. O., Natsuaki, R., Xu, X., Shimada, M., Hashimoto, M., Melgar, D., \& Sandwell, D. T. (2015). Line-of-sight displacement from alos-2 interferometry: Mw 7.8 gorkha earthquake and mw 7.3 aftershock. Geophysical Research Letters, 42(16), 6655-6661.

Lohman, R. B., \& Simons, M. (2005). Some thoughts on the use of insar data to constrain models of surface deformation: Noise structure and data downsampling. Geochemistry, Geophysics, Geosystems, 6(1).

López-Quiroz, P., Doin, M.-P., Tupin, F., Briole, P., \& Nicolas, J.-M. (2009). Time series analysis of mexico city subsidence constrained by radar interferometry. Journal of Applied Geophysics, 69(1), 1-15.

Massonnet, D., Rossi, M., Carmona, C., Adragna, F., Peltzer, G., Feigl, K., \& Rabaute, T. (1993). The displacement field of the landers earthquake mapped by radar interferometry. Nature, $364(6433), 138$.

Okada, Y. (1992). Internal deformation due to shear and tensile faults in a halfspace. Bulletin of the seismological society of America, 82(2), 1018-1040.

Palano, M., Aloisi, M., Amore, M., Bonforte, A., Calvagna, F., Cantarero, M., ... others (2006). Kinematics and strain analyses of the eastern segment of the pernicana fault (mt. etna, italy) derived from geodetic techniques (1997-2005). Annals of Geophysics, 49(4/5), 1105-1117.

Pritchard, M., \& Simons, M. (2004). An insar-based survey of volcanic deformation in the central andes. Geochemistry, Geophysics, Geosystems, 5(2).

Rosen, P. A., Hensley, S., Peltzer, G., \& Simons, M. (2004). Updated repeat orbit interferometry package released. Eos, Transactions American Geophysical Union, $85(5), 47-47$.

Rousset, B., Jolivet, R., Simons, M., Lasserre, C., Riel, B., Milillo, P., .. Renard, F. (2016). An aseismic slip transient on the north anatolian fault. Geophysical Research Letters, 43(7), 3254-3262.

Scheingross, J. S., Minchew, B. M., Mackey, B. H., Simons, M., Lamb, M. P., \& Hensley, S. (2013). Fault-zone controls on the spatial distribution of slow-moving landslides. Bulletin, 125(3-4), 473-489.

Schmidt, D. A., \& Bürgmann, R. (2003). Time-dependent land uplift and subsidence in the santa clara valley, california, from a large interferometric synthetic aperture 
radar data set. Journal of Geophysical Research: Solid Earth, 108(B9).

Segall, P., \& Matthews, M. (1997). Time dependent inversion of geodetic data. Journal of Geophysical Research: Solid Earth, 102(B10), 22391-22409.

Shirzaei, M., \& Bürgmann, R. (2018). Global climate change and local land subsidence exacerbate inundation risk to the san francisco bay area. Science advances, $4(3)$, eaap9234.

Shirzaei, M., \& Walter, T. (2010). Time-dependent volcano source monitoring using insar time series: a combined genetic algorithm and kalman filter approach. $J$. Geophys. Res, 115, B10421.

Simons, M., \& Rosen, P. $\quad$ (2015, 01). Interferometric synthetic aperture radar geodesy. In G. Schubert (Ed.), (p. 339-385). Elsevier. doi: 10.1016/ B978-0-444-53802-4.00061-0

Sudhaus, H., \& Jónsson, S. (2009). Improved source modelling through combined use of insar and gps under consideration of correlated data errors: application to the june 2000 kleifarvatn earthquake, iceland. Geophysical Journal International, $176(2), 389-404$.

Tarantola, A. (2005). Inverse problem theory and methods for model parameter estimation (Vol. 89). SIAM.

Tong, X., \& Schmidt, D. (2016). Active movement of the cascade landslide complex in washington from a coherence-based insar time series method. Remote Sensing of Environment, 186, 405-415.

Usai, S. (2003). A least squares database approach for sar interferometric data. IEEE Transactions on Geoscience and Remote Sensing, 41(4), 753-760.

Zebker, H. A., \& Villasenor, J. (1992). Decorrelation in interferometric radar echoes. IEEE Transactions on geoscience and remote sensing, 30(5), 950-959. 


\section{Appendix}

\subsection{Explicit formulation of an example}

To explicitly present our Kalman filter (Equations 3, 4 and 5) and the design of each matrix for InSAR data (Table 1), we describe an example below. We consider the case of the Kalman filter at the 2nd assimilation of data $(k=2)$ for a linear phase model, with an offset and a velocity $\phi_{i}=a_{0}+a_{1} t_{i}$. The state vector, then, writes as $\mathbf{m}_{1}=\left(a_{0}, a_{1}, \phi_{0}, \phi_{1}\right)$. After assimilation of data at time $t_{1}$, we have the covariance $\mathbf{P}_{1}$, the measurement $\mathbf{A}_{2}$ and the noise $\mathbf{Q}_{2}$ as

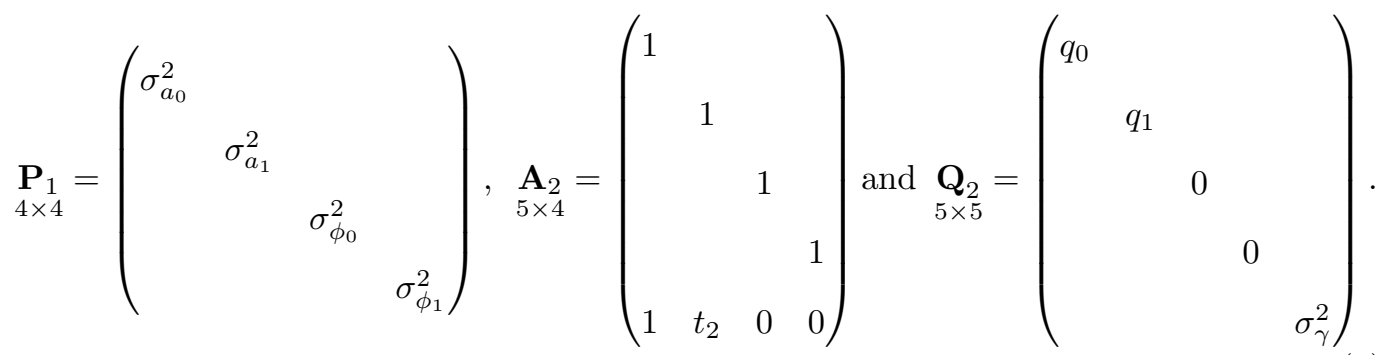

Because we want to exactly reconstruct phases with respect to a fixed null starting phase, $\phi_{0}$, then $\sigma_{\phi_{0}}$ must be set to zero. The parameters $q_{0}$ and $q_{1}$ are non-zero if there is a need to add systematic noise for functional parameters $a_{0,1}$. This would relax the weight of the previous estimate of $a_{0,1}$ on each forecast.

We consider two interferograms, $\phi_{2}-\phi_{0}$ and $\phi_{2}-\phi_{1}$, from 3 acquisitions at times $t_{0}$, $t_{1}$ and $t_{2}$. Thus the data, observation model $\mathbf{H}_{2}$ and covariance $\mathbf{R}_{2}$ are given as

$$
\underset{1 \times 2}{\mathbf{d}_{2}}=\left(\Phi_{02}, \Phi_{12}\right), \underset{2 \times 5}{\mathbf{H}_{2}}=\left(\begin{array}{ccccc}
0 & 0 & 0 & 0 & 1 \\
0 & 0 & 0 & -1 & 1
\end{array}\right) \text { and } \underset{2 \times 2}{\mathbf{R}_{2}}=\left(\begin{array}{cc}
\sigma_{\epsilon}^{2} & 0 \\
0 & \sigma_{\epsilon}^{2}
\end{array}\right)
$$

Applying Equations 3,

$$
\sigma_{\phi_{2}}^{f}=\sqrt{\sigma_{a_{0}}^{2}+\sigma_{a_{1}}^{2} t_{2}^{2}+\sigma_{\gamma}^{2}} \text {. and } \phi_{2}^{f}=a_{0}+a_{1} t_{2}
$$

Note that, if data is sufficient, the phase $\phi_{1}$ would have been reconstructed at the previous step with little uncertainty, so that $\sigma_{\phi_{1}} \rightarrow 0$. Following this assumption and using the data in $d_{2}$, we update the forecast with Equations 4 and 5. As an example, we have 


$$
\begin{gathered}
\left(\sigma_{\phi_{2}}\right)_{k=2}=\sqrt{\left(\sigma_{\phi_{2}}^{f}\right)^{2}-2 \kappa\left(\sigma_{\phi_{2}}^{f}\right)^{4}} \text { and }\left(\phi_{2}\right)_{k=2}=\phi_{2}^{f}+\rho \kappa\left(\sigma_{\phi_{2}}^{f}\right)^{2} \\
\left(\sigma_{a_{1}}\right)_{k=2}=\sqrt{\sigma_{a_{1}}^{2}-2 \kappa \sigma_{a_{1}}^{4} t_{2}^{2}+q_{0}} \text { and }\left(a_{1}\right)_{k=2}=a_{1}+\rho \kappa \sigma_{a_{1}}^{2} t_{2}
\end{gathered}
$$

with $\kappa$ the common part of the gain to all analyzed parameters and $\rho$ the residual expressed as

$$
\kappa=\frac{1}{\sigma_{\epsilon}^{2}+2\left(\sigma_{\phi_{2}}^{f}\right)^{2}} \quad \text { and } \quad \rho=\Phi_{02}+\Phi_{12}+\left(\phi_{1}\right)_{k=1}-2 \phi_{2}^{f}
$$

The subscript ' $\mathrm{k}=2$ ' outlines that the values are those evaluated at the second assimilation step. The velocity $a_{1}$ will be re-analyzed at each assimilation step and the phase $\phi_{2}$ may be re-analyzed if interferogram(s) $\Phi_{2 k}$ for any $k$ are assimilated over the course of subsequent assimilation steps. If noise associated with interferogram construction is small (i.e. $\sigma_{\epsilon} \rightarrow 0$ ), then Equation 12 tells us that the phase at time $t_{2}$ is perfectly reconstructed with zero uncertainty. In a more general sens, Equations 12 and 13 evidence the dependency of any phase and model parameter estimate to error terms arising from governing Equations 3, 4 and 5 (see Figures 11). 